\title{
The Complementary Exponentiated Lomax-Poisson Distribution with Applications to Bladder Cancer and Failure Data
}

\author{
Devendra Kumar \\ Central University of Haryana
}

\author{
Mazen Nassar
}

King Abdulaziz University

\author{
Ahmed Z. Afify \\ Benha University
}

\author{
Sanku Dey \\ St. Anthony's College
}

\begin{abstract}
A new continuous four-parameter lifetime distribution is introduced by compounding the distribution of the maximum of a sequence of an independently identically exponentiated Lomax distributed random variables and zero truncated Poisson random variable, defined as the complementary exponentiated Lomax Poisson (CELP) distribution. The new distribution which exhibits decreasing and upside down bathtub shaped density while the distribution has the ability to model lifetime data with decreasing, increasing and upside-down bathtub shaped failure rates. The new distribution has a number of well-known lifetime special sub-models, such as Lomax-zero truncated Poisson distribution, exponentiated Pareto-zero truncated Poisson distribution and Pareto- zero truncated Poisson distribution. A comprehensive account of the mathematical and statistical properties of the new distribution is presented. The model parameters are obtained by the methods of maximum likelihood, least squares, weighted least squares, percentiles, maximum product of spacing and Cramér-von-Mises and compared them using Monte Carlo simulation study. We illustrate the performance of the proposed distribution by means of two real data sets and both the data sets show the new distribution is more appropriate as compared to the transmuted Lomax, beta exponentiated Lomax, McDonald Lomax, Kumaraswamy Lomax, Weibull Lomax, Burr X Lomax and Lomax distributions.
\end{abstract}

Keywords: hazard rate function, maximum likelihood estimation, method of maximum product spacing, exponentiated Lomax Poisson, moments, Lorenz and Benferroni curves, order statistics.

\section{Introduction}

Over the last three decades or so, there has been an impetus in constructing new univariate distributions either due to the theoretical considerations or practical applications or both which are used widely in statistics and allied areas. Present trends and practices in defining probability distributions are different from those proposed before 1997. Now-a-days researchers have shown greater interest in defining new generators or generalized classes of univariate continuous distributions by adding one or more parameters or by compounding to generate new distributions to a baseline distribution to make the generated distribution more flexible, especially for studying tail behavior. Several authors have constructed dis- 
tributions by compounding the exponential with other discrete distributions. For example, (Adamidis and Loukas 1998) constructed the exponential geometric distribution by compounding the exponential distribution with a geometric distribution. (Kus 2007) proposed the exponential Poisson distribution by mixing an exponential and zero truncated Poisson distribution and discussed its various properties, (Barreto-Souza and Cribari-Neto 2009) discussed another generalized exponential-Poisson distribution by inserting a power parameter in exponential-Poisson distribution, (Cancho, Louzada-Neto, and Barriga 2011) introduced the two-parameter Poisson-exponential lifetime distribution with increasing failure rate, (Lu and Shi 2012) presented the Weibull-Poisson distribution as generalization of exponential-Poisson distribution, (Mahmoudi and Sepahdar 2013) introduced exponentiated Weibull-Poisson distribution and discussed its properties, (Louzada, Marchi, and Carpenter 2013) proposed complementary exponentiated exponential geometric distribution and (Ristic and Nadarajah 2014) introduced exponentiated exponential Poisson distribution. Recently, (Tahir and Cordeiro 2016) reviewed more than 20 already introduced distributions based on the zero truncated Poisson distribution.

In this paper we introduce a new lifetime distribution by compounding exponentiated Lomax distribution and zero truncated Poisson distribution, referred to as complementary exponentiated Lomax Poisson (CELP) distribution. The proposed distribution encompasses the behavior of some well known lifetime distributions and provides better fits than them, such as Lomax (Lomax 1954), transmuted Lomax (Ashour and Eltehiwy 2013), McDonald Lomax and Kumaraswamy Lomax (Lemonte and Cordeiro 2013), Weibull Lomax (Tahir, Cordeiro, Mansoor, and Zubair 2015), beta exponentiated Lomax (Mead 2016) and Burr X Lomax (Yousof, Afify, Hamedani, and Aryal 2017) distributions. We are motivated to introduce the CELP distribution because (i) it is capable of modeling decreasing, increasing and upsidedown bathtub shaped hazard rates; (ii) it can be viewed as a suitable model for fitting the skewed data which may not be properly fitted by other common distributions and can also be used in a variety of problems in different areas such as industrial reliability and survival analysis; and (iii) two real data applications show that it compares well with other competing lifetime distributions in modeling survival and failure data. A simple interpretation of the proposed model comes from a situation where failure (of a device for example) occurs due to the presence of an unknown number, say $Z$, of initial defects of the same kind. $Z$ follows a Poisson variable. Their lifetimes, $Y^{\prime} s$, follow an exponentiated Lomax distribution. Then for modeling the maximum failure $X$, the distribution leads to the complementary exponentiated Lomax-Poisson distribution. This distribution is a suitable model in a complementary risk problem (Basu and Klein 1982) in presence of latent risks, in a sense that there is no information about which factor was responsible for the component failure and only the maximum lifetime value among all risks is observed.

The motivation of the paper is two fold: first is to study the properties of the CELP distribution, and second is to estimate the parameters of the model using six frequentist methods of estimation, namely, methods of maximum likelihood, least squares, weighted least squares, percentiles, maximum product of spacing and Cramér-von-Mises for different sample sizes and different parameter values and to develop a guideline for choosing the best estimation method that gives better estimates for the CELP distribution, which we think would be of deep interest to applied statisticians. Comparisons of different estimation methods were compared for generalized Rayleigh distributions by (Kundu and Raqab 2005); for generalized logistic distributions by (Alkasasbeh and Raqab 2009); for weighted Lindley distribution by (Mazucheli, Louzada, and Ghitany 2013); for transmuted extended exponential distribution by (Kumar and Kumar 2019); for the two-parameter Rayleigh, weighted exponential, two-parameter Maxwell, exponentiated-Chen, two parameter exponentiated-Gumbel, new extension of generalized exponential and Nadarajah-Haghighi distributions by (Dey, Dey, and D. 2014), (Dey, Ali, and Park 2015), (Dey, Dey, Ali, and Mulekar 2016), (Dey, Alzaatreh, Zhang, and Ku- 
mar 2017a), (Dey, Kumar, Ramos, and Louzada 2017b), (Dey, Raheem, Mukherjee, and Ng 2017c), (Dey, Zhang, Asgharzadeh, and Ghorbannezhad 2017d), and for alpha logarithmic transformed Weibull, quasi xgamma-geometric, Weibull Marshall-Olkin Lindley, extended odd Weibull exponential, alpha power exponential distributions by (Nassar, Afify, Dey, and Kumar 2018a), (Sen, Afify, Al-Mofleh, and Ahsanullah 2019), (Afify, Nassar, Cordeiro, and Kumar 2020), (Afify and Mohamed 2020) and (Nassar, Afify, and Shakhatreh 2020).

The article is organized as follows. In the next section, we introduce the CELP distribution. Some mathematical and statistical properties of the CELP distribution are presented in Section 3. In Section 4, different frequentist methods of estimation are discussed. Monte Carlo simulation study is carried out to compare the different methods of estimation in Section 5. The potentiality of the new model is illustrated by means of application to real data in Section 6. Finally, some concluding remarks are addressed in Section 7.

\section{The CELP distribution}

(Lomax 1954) introduced a lifetime distribution, called Lomax (Pareto II) distribution. It is quite useful to analyze data related to business failure, income distribution and several other fields. The Lomax distribution with parameters $\alpha>0$ and $\beta>0$, say $\operatorname{Lomax}(\alpha, \beta)$, is given by the cumulative distribution function $(c d f)$

$$
G(x ; \alpha, \beta)=1-(1+\beta x)^{-\alpha}, \quad x>0,
$$

where $\alpha>0$ and $\beta>0$ are the shape and scale parameters, respectively. The corresponding probability density function (pdf) is given by

$$
g(x ; \alpha, \beta)=\alpha \beta(1+\beta x)^{-(\alpha+1)}, \quad x>0 .
$$

The $r^{\text {th }}$ moment of Lomax distribution is given by

$$
E\left(X^{r}\right)=\alpha \beta^{-r} B(\alpha-r, 1+r),
$$

where $\mathrm{B}(.,$.$) denotes the beta function defined by B(a, b)=\int_{0}^{\infty} \frac{x^{a-1}}{(1+x)^{a+b}} d x$.

The Lomax distribution has been explored by several authors. Introducing new parameters such as location, scale, shape and inequality in the existing distributions in order to add more flexibility has been adopted by several authors in the last 30 years or so. (Abdul-Moniem and Abdel-Hameed 2012) introduced the exponentiated Lomax (EL) distribution and studied few of its properties. The cdf of the EL distribution with parameters $\alpha, \beta$, and $\theta$, say $\operatorname{EL}(\alpha, \beta, \theta)$, is given by

$$
G(x ; \alpha, \beta, \theta)=\left(1-(1+\beta x)^{-\alpha}\right)^{\theta}, \quad x>0, \alpha, \beta, \theta>0,
$$

and the corresponding pdf is given by

$$
g(x ; \alpha, \beta, \theta)=\alpha \beta \theta(1+\beta x)^{-(\alpha+1)}\left(1-(1+\beta x)^{-\alpha}\right)^{\theta-1}, \quad x>0, \alpha, \beta, \theta>0 .
$$

(Al-Awadhi and Ghitany 2001) proposed Poisson-Lomax distribution as a mixture of Lomax and Poisson distribution. (Abd-Ellah 2003) obtained Bayesian prediction bounds for certain order statistics from Lomax distribution and (Abd-Elfattah, Alaboud, and Alharby 2007) discussed the Bayesian and non-Bayesian estimation of the reliability of Lomax distribution. (Ghitany, Al-Awadhi, and Alkhalfan 2007) proposed a new model called Marshall-Olkin extended Lomax distribution with its application to censored data. (Hassan and Al-Ghamdi 2009) discussed the estimation under optimum step stress accelerated life testing for the Lomax distribution. (Ramos, Marinho, Silva, and Cordeiro 2013) introduced the exponentiated Lomax Poisson (ELP) distribution with an application to lifetime data. (Hassan and Nassr 2018) proposed the power Lomax Poisson distribution, (Nassar, Dey, and Kumar 2018b) studied logarithm transformed Lomax distribution, (Dey, Nassar, Kumar, Alzaatreh, and 
Tahir 2019) proposed the alpha power transformed Lomax distribution, (Cordeiro, Afify, Ortega, Suzuki, and Mead 2019) introduced the odd-Lomax Lomax and (Afify, Cordeiro, Maed, Alizadeh, Al-Mofleh, and Nofal 2019) introduced the generalized odd Lindley Lomax distribution.

Given $Z \in N$, let $Y_{1}, Y_{2}, \ldots, Y_{z}$ be independent and identically distributed (iid) random variables with $p d f$ given in (5). Let $Z$ be a discrete random variable, having zero-truncated Poisson (ZTP) distribution with probability mass function (pmf)

$$
P(Z ; \lambda)=\frac{\lambda^{z}}{\left(e^{\lambda}-1\right) z !}, \quad \lambda>0, \quad z=1,2,3, \ldots
$$

Suppose that $X$ is a random variable representing the lifetime of a parallel system of $Z$ components, i.e. $X=\max \left\{Y_{i}\right\}, i=1,2, \ldots, z$, and $Y^{\prime} s$ and $Z$ are independent. The conditional pdf of $X \mid Z$ can be written as

$$
f_{X \mid Z}(x \mid z)=z g(x)[G(x)]^{z-1},
$$

where $G($.$) and g($.$) are the cdf and pdf of the EL distribution, respectively. The p d f$ of $X$ is obtained as follows.

Remark 1. If $X=\min \left\{Y_{i}\right\}$, we have the ELP distribution proposed by (Ramos et al. 2013).

Proposition 1. Let $X=\max \left\{Y_{i}\right\}$, where $Y_{i}=E L(\alpha, \beta, \theta)$, then according to (6) and (7), $X$ is distributed as CELP distribution, given by

$$
f(x)=\frac{\alpha \beta \lambda \theta(1+\beta x)^{-(\alpha+1)}\left(1-(1+\beta x)^{-\alpha}\right)^{\theta-1} \exp \left[\lambda\left(1-(1+\beta x)^{-\alpha}\right)^{\theta}\right]}{\left(e^{\lambda}-1\right)},
$$

with parameters $\alpha, \beta, \theta$ and $\lambda>0$.

Proof. By considering (6) and (7), the pdf of CELP $(\alpha, \beta, \lambda, \theta)$ can be computed by simplifying the unconditional pdf of $X$, given by

$$
f(x)=\sum_{z=1}^{\infty} f(x \mid z) P(Z=z) .
$$

The corresponding cdf of CELP distribution is given by

$$
F(x)=\frac{\exp \left(\lambda\left(1-(1+\beta x)^{-\alpha}\right)^{\theta}\right)-1}{\left(e^{\lambda}-1\right)} .
$$

The survival function (sf) and hazard rate function (hrf) of the CELP distribution are, respectively, given by

$$
S(x)=\frac{\exp (\lambda)-\exp \left(\lambda\left(1-(1+\beta x)^{-\alpha}\right)^{\theta}\right)}{\left(e^{\lambda}-1\right)}
$$

and

$$
h(x)=\frac{\alpha \beta \lambda \theta(1+\beta x)^{-(\alpha+1)}\left(1-(1+\beta x)^{-\alpha}\right)^{\theta-1} \exp \left(\lambda\left(1-(1+\beta x)^{-\alpha}\right)^{\theta}\right)}{\exp (\lambda)-\exp \left(\lambda\left(1-(1+\beta x)^{-\alpha}\right)^{\theta}\right)} .
$$

The pdf, hrf of CELP distribution have been plotted in Figures 1 and 2, respectively, for different values of $\alpha, \beta, \theta$ and $\lambda$.

Here are some special cases of the CELP model: Let $X \sim \operatorname{CELP}(\alpha, \beta, \lambda, \theta)$.

1. If $\theta=1$, then $X$ reduces to the Lomax-ZTP distribution. 
2. If $\beta=1$, then $X$ reduces to exponentiated Pareto-ZTP distribution.

3. If $\beta=1=\theta$, then $X$ reduces to Pareto-ZTP distribution.

4. If $\lambda \rightarrow 0^{+}$and $\theta=1$, the CELP distribution reduces to the Lomax ditribution.

5. If $\lambda \rightarrow 0^{+}$and $\beta=1$, the CELP distribution reduces to the exponentiated Pareto ditribution.

6. If $\alpha=1$, the CELP distribution reduces to the complementary Lomax Poisson distribution.
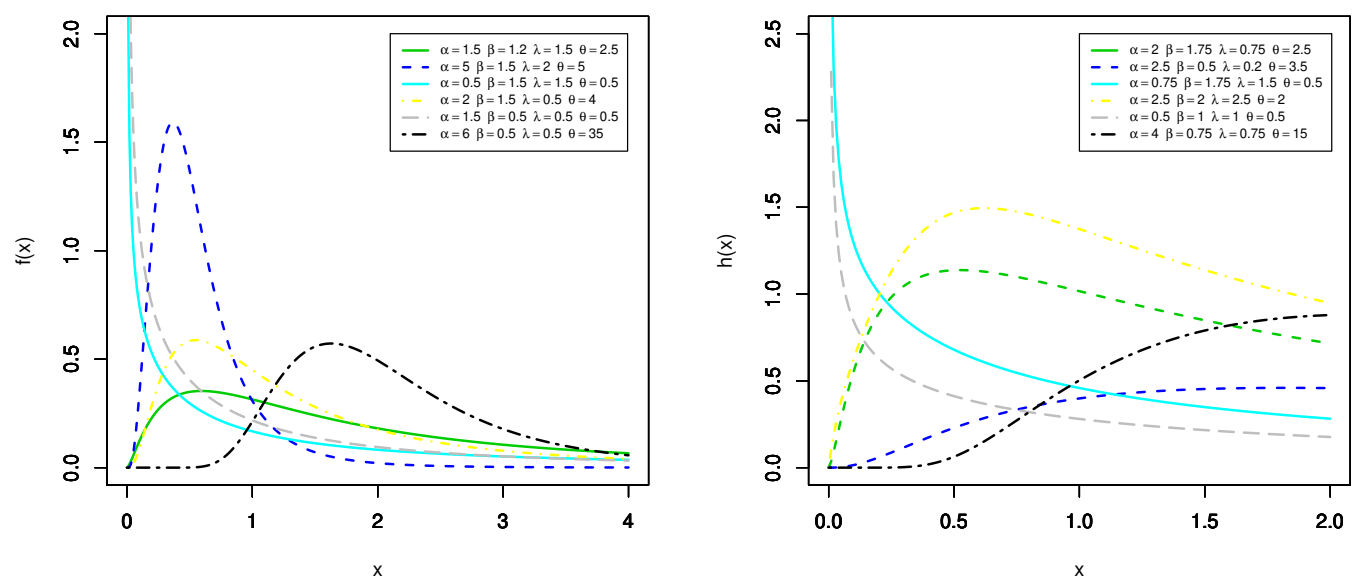

Figure 1: The pdf and hazard rate function plots of the CELP distribution for selected parameter values

Theorem 1. The limiting distribution given by (9) when $\lambda \rightarrow 0^{+}$is $\lim _{\lambda \rightarrow 0^{+}} F(x ; \alpha, \beta, \lambda, \theta)=$ $\left(1-(1+\beta x)^{-\alpha}\right)^{\theta}$, which is the cdf of $E L(\alpha, \beta, \theta)$.

Corollary 1. The pdf and hrf of the CELP distribution can be expressed in terms of the cdf and pdf of the EL distribution as

$$
f(x)=\omega g(x) e^{\lambda G(x)}
$$

and

$$
h(x)=\frac{\lambda g(x) e^{\lambda G(x)}}{e^{\lambda}-e^{\lambda G(x)}},
$$

repectively, where $\omega=\lambda\left(e^{\lambda}-1\right)^{-1} . G(x)$ and $g(x)$ are the $c d f$ and $p d f$ of the $E L$ distribution, given by (4) and (5), respectively.

\subsection{Series expansion of CELP distribution}

Here we express the pdf of CELP distribution as an infinite series of EL distribution or Lomax distribution. Using the exponential expansion of $\exp \left(\lambda\left(1-(1+\beta x)^{-\alpha}\right)^{\theta}\right.$ in (8),

$$
f(x)=\sum_{i=0}^{\infty} \psi_{i} g(x ; \alpha, \beta, \theta(i+1)),
$$

where

$$
\psi_{i}=\frac{\lambda^{i+1}}{\left(e^{\lambda}-1\right)(i+1) i !},
$$


and $g(x ; \alpha, \beta, \theta(i+1))$ is an EL distribution with parameters $\alpha, \beta$ and $\theta(i+1)$. Also for $b>0$ real and non-integer, $|a|<1$,

$$
(1-a)^{b-1}=\sum_{j=0}^{\infty} \frac{(-1)^{j} \Gamma(b) a^{j}}{\Gamma(b-j) j !} .
$$

For $\theta(i+1)>0$ real and non-integer, we can apply (14) in the expansion of $(1-(1+$ $\left.\beta x)^{-\alpha}\right)^{\theta(i+1)-1}$ in (12) and after some algebraic manipulations, we obtain

$$
f(x)=\sum_{i=0}^{\infty} \sum_{j=0}^{\infty} \xi_{i, j} g(x ; \alpha, \beta(j+1))
$$

where

$$
\xi_{i, j}=\frac{(-1)^{j} \theta \lambda^{i+1} \Gamma(\theta(i+1))}{\left(e^{\lambda}-1\right)(j+1) \Gamma(\theta(i+1)-j) i ! j !}
$$

and $g(x ; \alpha, \beta(j+1))$ is Lomax distribution with parameters $\alpha$ and $\beta(j+1)$.

\section{Statistical and mathematical properties}

In this section, we devoted to some statistical and mathematical properties of the CELP distribution.

\subsection{Quantile function}

The quantile function are in widespread use in general statistics to obtain mathematical properties of a distribution and often find the representation in terma of lookup tables for key percentiles. For generating data from the CELP model, let $p \sim U(0,1)$. Then, by inverting the cdf (9) and after some algebra, we get the quantile function

$$
x_{p}=Q(p)=\frac{1}{\beta}\left[\left\{1-\left(\frac{\log \left(p\left(e^{\lambda}-1\right)+1\right)}{\lambda}\right)^{1 / \theta}\right\}^{-1 / \alpha}-1\right], \quad 0<p<1 .
$$

In particular, the first three quantiles, $Q_{1}, Q_{2}$ and $Q_{3}$, can be obtained by setting $p=0.25$, $p=0.5$ and $p=0.75$ in equation (16) respectively. Note that $x_{p}$ can be used to generate CELP random variates.

The analysis of variability of the skewness and kurtosis of $X$ can be investigated based on quentile measure the Bowley skewness is given by

$$
B=\frac{Q(3 / 4)+Q(1 / 4)-2 Q(1 / 2)}{Q(3 / 4)-Q(1 / 4)}
$$

and Moors' kurtosis (Moors 1988) by

$$
M=\frac{Q(3 / 8)-Q(1 / 8)+Q(7 / 8)-Q(5 / 8)}{Q(6 / 8)-Q(2 / 8)},
$$

where $Q($.$) is a quantile function given by (16).$

These measures are less sensitive to outliers and they exit even for distributions without moments. Figure 2 display plots of $B$ and $M$ as function of $\alpha, \beta$ and $\theta$, which shows their variability in terms of the shape parameters. 

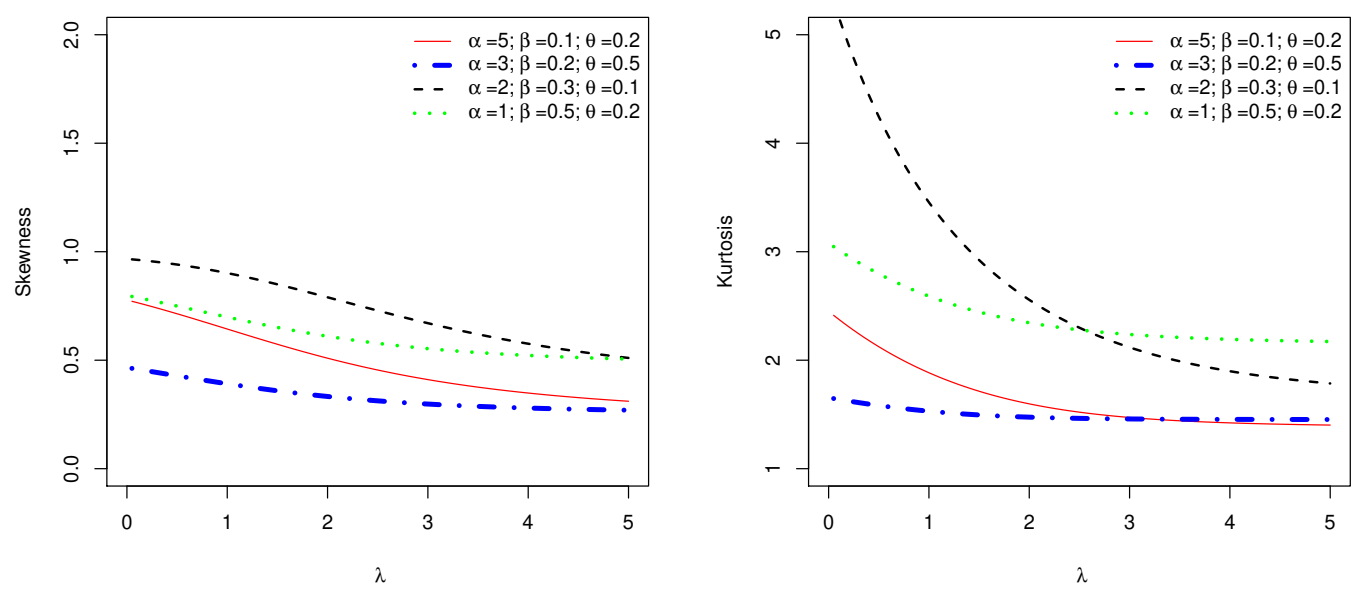

Figure 2: Plots of B-skewness and M-kurtosis of CELP distribution for different parameter values

\subsection{Moments and moment generating function}

The moments, incomplete moments, moment generating function, skewness and kurtosis of a probability distribution are very important tools to illustrate the distribution. Let the random variable $Y$ follow the lomax distribution with $\alpha>0$ and $\beta>0(\operatorname{Lomax}(\alpha, \beta))$. Then

$$
g(y ; \alpha, \beta)=\alpha \beta(1+\beta y)^{-(\alpha+1)}, \quad y>0 .
$$

(Lomax 1954) showed that

$$
E\left(Y^{r}\right)=\alpha \beta^{-r} B(\alpha-r, 1+r),
$$

Now, the $r^{\text {th }}$ moment of CELP distribution can be written as

$$
\begin{aligned}
E\left(X^{r}\right) & =\int_{0}^{\infty} x^{r} f(x) d x \\
& =\sum_{i=0}^{\infty} \sum_{j=0}^{\infty} \xi_{i, j} \int_{0}^{\infty} x^{r} \alpha \beta(j+1)(1+\beta(j+1) x)^{-\alpha-1} d x \\
& =\sum_{i=0}^{\infty} \sum_{j=0}^{\infty} \xi_{i, j} E\left[Y^{r}\right]
\end{aligned}
$$

where $Y \sim \operatorname{Lomax}(\alpha, \beta(j+1))$ is the pdf of Lomax distribution with parameters $\alpha$ and $\beta(j+1)$. On using (17) we get

$$
E\left(X^{r}\right)=\sum_{i=0}^{\infty} \sum_{j=0}^{\infty} \xi_{i, j, r} B(\alpha-r, 1+r),
$$

where

$$
\xi_{i, j, r}=\frac{(-1)^{j} \alpha \theta \lambda^{i+1} \beta^{-r} \Gamma(\theta(i+1))}{\left(e^{\lambda}-1\right)(j+1)^{r+1} \Gamma(\theta(i+1)-j) i ! j !} .
$$

The skewness $\left(\gamma_{1}\right)$ and kurtosis $\left(\gamma_{2}\right)$ of the CELP distribution are, respectively, obtained from

$$
\gamma_{1}=\frac{1}{\sigma^{3}} \sum_{r=0}^{3}\left(\begin{array}{l}
3 \\
r
\end{array}\right)(-1)^{r+1} \mu^{3-r} E\left(X^{r}\right)
$$


and

$$
\gamma_{2}=\frac{1}{\sigma^{4}} \sum_{r=0}^{4}\left(\begin{array}{l}
4 \\
r
\end{array}\right)(-1)^{r} \mu^{4-r} E\left(X^{r}\right)
$$

Using (18), we obtain

$$
\gamma_{1}=\sum_{r=0}^{3} \sum_{i=0}^{\infty} \sum_{j=0}^{\infty} \frac{(-1)^{j+r+1}\left(\begin{array}{l}
3 \\
r
\end{array}\right) \alpha \theta \lambda^{i+1} \beta^{-r} \mu^{3-r} \Gamma(\theta(i+1))}{\sigma^{3}\left(e^{\lambda}-1\right)(j+1)^{r+1} \Gamma(\theta(i+1)-j) i ! j !} B(\alpha-r, 1+r),
$$

and

$$
\gamma_{2}=\sum_{r=0}^{4} \sum_{i=0}^{\infty} \sum_{j=0}^{\infty} \frac{(-1)^{j+r}\left(\begin{array}{l}
4 \\
r
\end{array}\right) \alpha \theta \lambda^{i+1} \beta^{-r} \mu^{4-r} \Gamma(\theta(i+1))}{\sigma^{4}\left(e^{\lambda}-1\right)(j+1)^{r+1} \Gamma(\theta(i+1)-j) i ! j !} B(\alpha-r, 1+r),
$$

where $\mu$ and $\sigma$ are the mean and standard deviation of the CELP distribution.

The relations in (18) can be used to compute the expected values and higher order moments of the CELP distribution. In Table 1, we have presented the expected values, variances, skewness and kurtosis of the CELP distribution for $\lambda=1.1,1.2, \ldots, 2.0$ and $\theta=1.1,1.2, \ldots, 2.0$, respectively. One can see from Table 1 that the the means and variances are increasing with respect to $\lambda$ but skewness and kurtosis are decreasing with respect to $\lambda$. Similarly, the means and variances are increasing with respect to $\theta$ but skewness and kurtosis are decreasing with respect to $\theta$.

The moment generating function of CELP distribution can be computed by substituting (18) in $(21)$ as follows:

$$
\begin{aligned}
M_{x}(t) & =E\left(e^{t x}\right)=\sum_{r=0}^{\infty} \frac{t^{r}}{r !} E\left(X^{r}\right) \\
& =\sum_{r=0}^{\infty} \sum_{i=0}^{\infty} \sum_{j=0}^{\infty} \xi_{i, j, r}^{*} B(\alpha-r, 1+r),
\end{aligned}
$$

where

$$
\xi_{i, j, r}^{*}=\frac{(-1)^{j} \alpha \theta \lambda^{i+1} \beta^{-r} t^{r} \Gamma(\theta(i+1))}{\left(e^{\lambda}-1\right)(j+1)^{r+1} \Gamma(\theta(i+1)-j) i ! j ! r !} .
$$

Table 1: Expected values, variances, skewness and kurtosis of the CELP distribution for different values of parameters

\begin{tabular}{llllllllll}
\hline \multicolumn{4}{c}{$\alpha=5, \beta=2.5, \theta=1.5$} & \multicolumn{5}{c}{$\alpha=5, \beta=2.5, \lambda=1.5$} \\
\hline$\lambda$ & $\mathrm{E}(\mathrm{X})$ & $\mathrm{V}(\mathrm{X})$ & $\gamma_{1}$ & $\gamma_{2}$ & $\theta$ & $\mathrm{E}(\mathrm{X})$ & $\mathrm{V}(\mathrm{X})$ & $\gamma_{1}$ & \multicolumn{1}{c}{$\gamma_{2}$} \\
\hline 1.1 & 0.16810 & 0.02854 & 1.38110 & 51.75985 & 1.1 & 0.15314 & 0.02649 & 1.52053 & 53.08159 \\
1.2 & 0.17163 & 0.02921 & 1.35126 & 51.10784 & 1.2 & 0.161 & 0.02775 & 1.44479 & 51.96525 \\
1.3 & 0.17517 & 0.02987 & 1.32356 & 50.54843 & 1.3 & 0.16845 & 0.02894 & 1.37889 & 51.00714 \\
1.4 & 0.17872 & 0.03052 & 1.29586 & 49.99448 & 1.4 & 0.17552 & 0.03008 & 1.32061 & 50.19443 \\
1.5 & 0.18227 & 0.03117 & 1.26946 & 49.48087 & 1.5 & 0.18227 & 0.03117 & 1.26946 & 49.48087 \\
1.6 & 0.18582 & 0.03180 & 1.24428 & 49.02240 & 1.6 & 0.18872 & 0.03220 & 1.22386 & 48.85617 \\
1.7 & 0.18937 & 0.03244 & 1.21963 & 48.55791 & 1.7 & 0.1949 & 0.03319 & 1.18312 & 48.32825 \\
1.8 & 0.19291 & 0.03307 & 1.19609 & 48.14234 & 1.8 & 0.20083 & 0.03415 & 1.14613 & 47.85178 \\
1.9 & 0.19645 & 0.03368 & 1.17306 & 47.78042 & 1.9 & 0.20654 & 0.03507 & 1.11246 & 47.40381 \\
2.0 & 0.19998 & 0.03429 & 1.15101 & 47.41709 & 2.0 & 0.21205 & 0.03595 & 1.08183 & 47.02954 \\
\hline
\end{tabular}

\subsection{Conditional moment and mean deviation}

Here, we introduce an important lemma which will be used in the next section. 
Lemma 1. Let $X$ be a random variable with pdf given in (15) and let $J_{n}(t)=\int_{0}^{t} x^{n} f(x) d x$. Consider the lower incoimplete gamma function $\Gamma(t, a)=\int_{0}^{t} x^{a-1} e^{-x} d x$. Then we have

$$
J_{n}(t)=\frac{\alpha \theta}{\left(e^{\lambda}-1\right) \beta} \sum_{i=0}^{\infty} \sum_{j=0}^{\infty} \sum_{s=0}^{n} \frac{(-1)^{n+j+s} \lambda^{i+1}\left(\begin{array}{l}
n \\
s
\end{array}\right)\left[1-(1+\beta(j+1) t)^{(s-\alpha)}\right] \Gamma(\theta(i+1))}{(j+1)^{n+2} \Gamma(\theta(i+1)-j) i ! j !(\alpha-s)} .
$$

Proof. Using the equation (15), we have

$$
\begin{aligned}
J_{n}(t) & =\int_{0}^{t} x^{n} f(x) d x=\sum_{i=0}^{\infty} \sum_{j=0}^{\infty} \xi_{i, j} \int_{0}^{t} x^{n} g(x ; \alpha, \beta(j+1)) d x \\
& =\alpha \beta \sum_{i=0}^{\infty} \sum_{j=0}^{\infty} \xi_{i, j} \int_{0}^{t} x^{n}(1+\beta(j+1) x)^{-(\alpha+1)} d x \\
& =\frac{\alpha}{\beta} \sum_{i=0}^{\infty} \sum_{j=0}^{\infty} \sum_{s=0}^{n} \xi_{i, j}(-1)^{n+s}\left(\begin{array}{c}
n \\
s
\end{array}\right) \frac{\left[1-(1+\beta(j+1) t)^{(s-\alpha)}\right]}{(j+1)^{n+1}(\alpha-s)}
\end{aligned}
$$

The proof is complete.

The $n$-th conditional moments of the CELP distribution is given by

$$
\eta_{n}(t)=E\left[X^{n} \mid x>t\right]=\frac{1}{1-F(t)} \int_{t}^{\infty} x^{n} f(x) d x=\frac{1}{S(t)}\left[E\left(X^{n}\right)-J_{n}(t)\right] .
$$

It can be expressed using (10), (18) and Lemma 1 . The same remark hold for the $n$-th reversed moments of the CELP distribution is given by

$$
m_{n}(t)=E\left[X^{n} \mid x \leq t\right]=\frac{1}{F(t)} \int_{0}^{t} x^{n} f(x) d x=\frac{1}{F(t)} J_{n}(t) .
$$

The important application of the first reversed moment is related to Bonferroni and Lorenz curves defined by $L(p)=m_{1}\left(x_{p}\right) / \mu_{1}^{\prime}$ and $B(p)=m_{1}\left(x_{p}\right) / p \mu_{1}^{\prime}$, respectively, where $x_{p}$ can be evaluated numerically from equation (16) for a given probability. These curves are very useful in economics, demography, insurance, engineering and medicine.

\subsection{Entropies}

An entropy can be considered as a measeure of uncertainty of probability distribution of a random variable. Therefore, we obtain two entropies for CELP distribution.

Entropy 1. Let us now focus our attention on the Rényi entropy. If $X$ has the probability distribution function $f(\cdot)$, Rényi entropy is defined as

$$
\Im_{R}(\delta)=\frac{1}{1-\delta} \log \left(\int_{0}^{\infty} f^{\delta}(x) d x\right), \quad \delta>0, \quad \delta \neq 1 .
$$

Using equation (8), we have

$$
f^{\delta}(x)=\left(\frac{\alpha \beta \lambda \theta}{e^{\lambda}-1}\right)^{\delta}(1+\beta x)^{-\delta(\alpha+1)}\left[1-(1+\beta x)^{-\alpha}\right]^{\delta(\theta-1)} e^{\delta \lambda\left[1-(1+\beta x)^{-\alpha}\right]^{\theta}} .
$$

After some algebra, we can write

$$
f^{\delta}(x)=\left(\frac{\alpha \beta \lambda \theta}{e^{\lambda}-1}\right)^{\delta} \sum_{i=0}^{\infty} \sum_{j=0}^{\infty} \frac{(-1)^{j}(\delta \lambda)^{i} \Gamma[\delta(\theta-1)+\theta i+1](1+\beta x)^{-\delta(\alpha+1)-\alpha j}}{i ! j ! \Gamma[\delta(\theta-1)+\theta i+1-j]} .
$$

Then, the Rényi entropy of $\mathrm{X}$ reduces to

$$
\Im_{R}(\delta)=\left(\left(\frac{\alpha \beta \lambda \theta}{e^{\lambda}-1}\right)^{\delta} \sum_{i=0}^{\infty} \sum_{j=0}^{\infty} \frac{(-1)^{j}(\delta \lambda)^{i} \Gamma[\delta(\theta-1)+\theta i+1]}{i ! j ! \Gamma[\delta(\theta-1)+\theta i+1-j]} \int_{0}^{k} \frac{1}{(1+\beta x)^{\delta(\alpha+1)+\alpha j}} d x\right) .
$$


Finally, it can be expressed as

$$
\Im_{R}(\delta)=\frac{1}{1-\delta} \log \left[\left(\frac{\alpha \beta \lambda \theta}{e^{\lambda}-1}\right)^{\delta} \sum_{i=0}^{\infty} e_{i}\right],
$$

where

$$
e_{i}=\sum_{j=0}^{\infty} \frac{(-1)^{j}(\delta \lambda)^{i} \Gamma[\delta(\theta-1)+\theta i+1] B(1, \delta(\alpha+1)+\alpha j)}{i ! j ! \beta \Gamma[\delta(\theta-1)+\theta i+1-j]} .
$$

The $\delta$-entropy, say $H_{\delta}(x)$, is defined by

$$
H_{\delta}(x)=\frac{1}{\delta-1} \log \left[1-\int_{0}^{1} f^{\delta}(x) d x\right], \quad \delta>0, \quad \delta \neq 1,
$$

and then it follows from equation (23).

Entropy 2. We now focus our attention on the entropy which is proposed by (Mathai and Haubold 2008): $J_{M H}(\gamma)=\frac{1}{\gamma-1}\left(\int_{-\infty}^{\infty} f^{2-\gamma}(x) d x-1\right)$. Proceeding as for $\Im_{R}(\delta)$ with $2-\gamma$ instead of $\delta$, we obtain

$f^{2-\gamma}(x)=\left(\frac{\alpha \beta \lambda \theta}{e^{\lambda}-1}\right)^{2-\gamma} \sum_{i=0}^{\infty} \sum_{j=0}^{\infty} \frac{(-1)^{j}(\lambda(2-\gamma))^{i} \Gamma[(2-\gamma)(\theta-1)+\theta i+1](1+\beta x)^{-(2-\gamma)(\alpha+1)-\alpha j}}{i ! j ! \Gamma[(2-\gamma)(\theta-1)+\theta i+1-j]}$.

Hence

$$
\begin{aligned}
J_{M H}(\gamma) & =\frac{1}{\gamma-1}\left(\int_{-\infty}^{\infty} f^{2-\gamma}(x) d x-1\right) \\
& =\frac{1}{\gamma-1}\left[\left(\frac{\alpha \beta \lambda \theta}{e^{\lambda}-1}\right)^{2-\gamma} \sum_{i=0}^{\infty} \phi_{i}-1\right]
\end{aligned}
$$

where

$$
\phi_{i}=\sum_{j=0}^{\infty} \frac{(-1)^{j}(\lambda(2-\gamma))^{i} \Gamma[(2-\gamma)(\theta-1)+\theta i+1] B(1,(2-\gamma)(\alpha+1)+\alpha j)}{i ! j ! \beta \Gamma[(2-\gamma)(\theta-1)+\theta i+1-j]} .
$$

\subsection{Residual life function}

The residual life is described by the conditional random variable $R_{(t)}=X-t \mid X>t, t \geq 0$.

The sf of the residual lifetime $R_{(t)}$ of the CELP distribution is

$$
S_{R_{(t)}}(x)=\frac{S(x+t)}{S(t)}=\frac{e^{\lambda}-e^{\lambda\left[1-\{1+\beta(x+t)\}^{-\alpha}\right]^{\theta}}}{e^{\lambda}-e^{\lambda\left[1-(1+\beta t)^{-\alpha}\right]^{\theta}}}, \quad x>0
$$

and the associated cdf is

$$
F_{R_{(t)}}(x)=\frac{e^{\lambda\left[1-\{1+\beta(x+t)\}^{-\alpha}\right]^{\theta}}-e^{\lambda\left[1-(1+\beta t)^{-\alpha}\right]^{\theta}}}{e^{\lambda}-e^{\lambda\left[1-(1+\beta t)^{-\alpha}\right]^{\theta}}} .
$$

The corresponding pdf is given by

$$
f_{R_{(t)}}(x)=\frac{\alpha \beta \theta \lambda\{1+\beta(x+t)\}^{-\alpha-1}\left[1-\{1+\beta(x+t)\}^{-\alpha}\right]^{\theta-1} e^{\lambda\left[1-\{1+\beta(x+t)\}^{-\alpha}\right]^{\theta}}}{e^{\lambda}-e^{\lambda\left[1-(1+\beta t)^{-\alpha}\right]^{\theta}}} .
$$

Therefore, the hrf of $R_{(t)}$ is

$$
h_{R_{(t)}}(x)=\frac{\alpha \beta \theta \lambda\{1+\beta(x+t)\}^{-\alpha-1}\left[1-\{1+\beta(x+t)\}^{-\alpha}\right]^{\theta-1} e^{\lambda\left[1-\{1+\beta(x+t)\}^{-\alpha}\right]^{\theta}}}{e^{\lambda}-e^{\lambda\left[1-\{1+\beta(x+t)\}^{-\alpha}\right]^{\theta}}} .
$$


The mean residual life is defined as

$$
I(t)=E\left(R_{(t)}\right)=\frac{1}{S(t)} \int_{t}^{\infty} x f(x) d x-t=\frac{1}{S(t)}\left[E(X)-J_{1}(t)\right]-t,
$$

where $f(x)$ is given by (8), $S(t)$ is given by (10), $E(X)$ is given by (18) for $r=1$ and $J_{1}(t)$ is given by Lemma 1 .

Further, the variance residual life is given by

$$
\begin{aligned}
V(t) & =\operatorname{Var}\left(R_{(t)}\right)=\frac{2}{S(t)} \int_{t}^{\infty} x S(x) d x-2 t I(t)-[I(t)]^{2} \\
& =\frac{1}{S(t)}\left[E\left(X^{2}\right)-J_{2}(t)\right]-t^{2}-2 t I(t)[I(t)]^{2},
\end{aligned}
$$

where $E\left(X^{2}\right)$ is given by (18) for $r=2$ and $J_{2}(t)$ is given by Lemma 1 .

\subsection{Reversed residual life function}

The reversed residual life is described by the conditional random variable $\bar{R}_{(t)}=t-X \mid X \leq t$, $t \geq 0$. The sf of the reverse residual lifetime $\bar{R}_{(t)}$ of the CELP distribution is given by

$$
S_{\bar{R}_{(t)}}(x)=\frac{F(t-x)}{F(t)}=\frac{e^{\lambda\left[1-\{1+\beta(t-x)\}^{-\alpha}\right]^{\theta}}-1}{e^{\lambda\left[1-(1+\beta t)^{-\alpha}\right]^{\theta}}-1}, \quad x>0
$$

and the associated cdf is

$$
F_{\bar{R}_{(t)}}(x)=\frac{e^{\lambda\left[1-(1+\beta t)^{-\alpha}\right]^{\theta}}-e^{\lambda\left[1-\{1+\beta(t-x)\}^{-\alpha}\right]^{\theta}}-2}{e^{\lambda\left[1-(1+\beta t)^{-\alpha}\right]^{\theta}}-1} .
$$

The corresponding pdf is given by

$$
f_{\bar{R}_{(t)}}(x)=\frac{\alpha \beta \theta \lambda\{1+\beta(t-x)\}^{-\alpha-1}\left[1-\{1+\beta(t-x)\}^{-\alpha}\right]^{\theta-1} e^{\lambda\left[1-\{1+\beta(t-x)\}^{-\alpha}\right]^{\theta}}}{e^{\lambda\left[1-(1+\beta t)^{-\alpha}\right]^{\theta}-1}} .
$$

Therefore, the hrf of $\bar{R}_{(t)}$ is

$$
h_{\bar{R}_{(t)}}(x)=\frac{\alpha \beta \theta \lambda\{1+\beta(t-x)\}^{-\alpha-1}\left[1-\{1+\beta(t-x)\}^{-\alpha}\right]^{\theta-1} e^{\lambda\left[1-\{1+\beta(t-x)\}^{-\alpha}\right]^{\theta}}}{e^{\lambda\left[1-\{1+\beta(t-x)\}^{-\alpha}\right]^{\theta}-1}} .
$$

Moreover, the mean reversed residual life is defined as

$$
J(t)=E\left(\bar{R}_{(t)}\right)=t-\frac{1}{F(t)} \int_{0}^{t} x f(x) d x=t-\frac{J_{1}(t)}{F(t)},
$$

where $f(x)$ is given by $(8), F(t)$ is given by $(9)$ and $J_{1}(t)$ is given by Lemma 1 .

Also, the variance reversed residual life is given by

$$
\begin{aligned}
W(t) & =\operatorname{Var}\left(\bar{R}_{(t)}\right)=2 t J(t)-[J(t)]^{2}-\frac{2}{F(t)} \int_{0}^{t} x F(x) d x \\
& =2 t J(t)-[J(t)]^{2}-t^{2}+\frac{J_{2}(t)}{F(t)}
\end{aligned}
$$

where $J_{2}(t)$ is given by Lemma 1 .

\subsection{Order statistics}

Let $X_{1}, X_{2}, \ldots, X_{n}$ be a random sample from CELP distribution. Let $X_{1: n} \leq X_{2: n} \leq \ldots \leq$ $X_{n: n}$ be the order statistics from this random sample, then the $p d f f_{i: n}(x)$ of the $i^{t h}$ order statistic, for $i=1,2, \ldots, n$, is obtained as follows:

$$
f_{i: n}(x)=\frac{n !}{(i-1) !(n-i) !} f(x)(F(x))^{i-1}(1-F(x))^{n-i},
$$


where $f(x)$ and $F(x)$ are the pdf and cdf of the CELP distribution, respectively.

$$
f_{i: n}(x)=\sum_{l=0}^{n-i} \frac{n !(-1)^{l}}{(i-1) !(n-i-l) ! l !} f(x)(F(x))^{i+l-1} .
$$

Using the binomial expansion of $F(x)$ in (24), we have

$$
f_{i: n}(x)=\sum_{l=0}^{n-i} \sum_{k=0}^{i+l-1} \frac{(-1)^{i+2 l+k-1} n !(i+l-1) ! \exp \left(k \lambda\left(1-(1+\beta x)^{-\alpha}\right)^{\theta}\right) f(x)}{\left(e^{\lambda}-1\right)^{i+l-1}(i+l-k-1) !(n-i-l) !(i-1) ! l ! k !} .
$$

After some algebraic manipulations, we obtain

$$
f_{i: n}(x)=\sum_{l=0}^{n-i} \sum_{k=0}^{i+l-1} \phi_{k, l} f(x ; \alpha, \beta, \theta, \lambda(k+1)),
$$

where

$$
\phi_{k, l}=\frac{(-1)^{i+2 l+k-1} n !(i+l-1) ! \exp (\lambda(k+1)-1)}{\left(e^{\lambda}-1\right)^{i+l}(i+l-k-1) !(n-i-l) !(i-1) !(k+1) l ! k !}
$$

and $f(x ; \alpha, \beta, \theta, \lambda(k+1))$ is the $p d f$ of CELP distribution with parameters $\alpha, \beta, \theta$ and $\lambda(k+1)$. The $r^{\text {th }}$ moment of the $i^{t h}$ order statistic is given by

$$
\begin{aligned}
E\left(X_{i: n}^{r}\right) & =\int_{0}^{\infty} x^{r} f_{i: n}(x) d x \\
& =\sum_{l=0}^{n-i} \sum_{k=0}^{i+l-1} \phi_{k, l} \int_{0}^{\infty} x^{r} f(x ; \alpha, \beta, \theta, \lambda(k+1)) d x .
\end{aligned}
$$

Thus, by considering (18), we obtain

$$
E\left(X_{i: n}^{r}\right)=\sum_{l=0}^{n-i} \sum_{k=0}^{i+l-1} \sum_{p=0}^{\infty} \sum_{q=0}^{\infty} \phi_{k, l}^{*} B(\alpha-r, 1+r),
$$

where

$$
\phi_{k, l}^{*}=\frac{(-1)^{q+i+2 l+k-1} n ! \alpha \theta \lambda^{p+1} \beta^{-r}(k+1)^{p} \Gamma(\theta(p+1))(i+l-1) !}{\left(e^{\lambda}-1\right)^{i+l}(i+l-k-1) !(n-i-l) !(i-1) !(q+1)^{r+1} \Gamma(\theta(p+1)-q) l ! k ! p ! q !} .
$$

\section{Parameters estimation and simulation study}

In this section, we consider six frequentist methods of estimation to estimate the unknown parameters of the CELP distribution. The first choice to estimate the unknown parameters of the distribution is the maximum likelihood methods. Recently, many authors prefer to use other classical methods such as method of maximum product of spacing and method of Percentiles, because these methods have some advantages over the maximum likelihood method. Here, we study the estimation problem of the unknown parameters using different methods of estimation to recommend the best method that can be used to estimate the distribution parameters. It is not possible to compare the performance of the different estimators theoretically. For this reason, we perform a simulation study to compare the performance of the different estimators using the minimum root mean square error criterion.

\subsection{Method of maximum likelihood}

Let $x_{1}, x_{2}, \cdots, x_{n}$ be a random sample of size $n$ from CELP distribution, then the loglikelihood function corresponding to Eq.(8) is as follows 


$$
\begin{aligned}
L(\alpha, \beta, \theta, \lambda) & =n \log (\alpha \beta \lambda \theta)-n \log \left(e^{\lambda}-1\right)-(\alpha+1) \sum_{i=1}^{n} \log \left(1+\beta x_{i}\right) \\
& +(\theta-1) \sum_{i=1}^{n} \log \left[1-\left(1+\beta x_{i}\right)^{-\alpha}\right]+\lambda \sum_{i=1}^{n}\left[1-\left(1+\beta x_{i}\right)^{-\alpha}\right]^{\theta}
\end{aligned}
$$

The maximum likelihood estimators (MLEs) of the CELP distribution parameters $\alpha, \beta, \lambda$ and $\theta$ can be obtained by solving the following four normal equations

$$
\begin{gathered}
\frac{\partial L(\alpha, \beta, \theta, \lambda)}{\partial \alpha}=\frac{n}{\alpha}-\sum_{i=1}^{n} \log \left(\nu_{i}\right)+(\theta-1) \sum_{i=1}^{n} \frac{\log \left(\nu_{i}\right)}{\nu_{i}^{\alpha}-1}+\lambda \theta \sum_{i=1}^{n} \nu_{i}^{-\alpha} \log \left(\nu_{i}\right)\left[1-\nu_{i}^{-\alpha}\right]^{\theta-1} \\
\frac{\partial L(\alpha, \beta, \theta, \lambda)}{\partial \beta}=\frac{n}{\beta}-(\alpha+1) \sum_{i=1}^{n} \frac{x_{i}}{\nu_{i}}+\alpha(\theta-1) \sum_{i=1}^{n} \frac{x_{i} \nu_{i}^{-(\alpha+1)}}{1-\nu_{i}^{-\alpha}}+\lambda \alpha \theta \sum_{i=1}^{n} x_{i} \nu_{i}^{-(\alpha+1)}\left[1-\nu_{i}^{-\alpha}\right]^{\theta-1}, \\
\frac{\partial L(\alpha, \beta, \theta, \lambda)}{\partial \theta}=\frac{n}{\theta}+\sum_{i=1}^{n} \log \left[1-\nu_{i}^{-\alpha}\right]+\lambda \sum_{i=1}^{n}\left[1-\nu_{i}^{-\alpha}\right]^{\theta} \log \left(1-\nu_{i}^{-\alpha}\right)
\end{gathered}
$$

and

$$
\frac{\partial L(\alpha, \beta, \theta, \lambda)}{\partial \lambda}=\frac{n}{\lambda}-n\left(1-e^{-\lambda}\right)^{-1}+\sum_{i=1}^{n}\left[1-\nu_{i}^{-\alpha}\right]^{\theta}
$$

where $\nu_{i} \equiv \nu\left(\beta ; x_{i}\right)=1+\beta x_{i}$. The MLEs of $\alpha, \beta, \lambda$ and $\theta$ denoted by $\hat{\alpha}_{M L E}, \hat{\beta}_{M L E}, \hat{\lambda}_{M L E}$ and $\hat{\theta}_{M L E}$ are obtained by solving the above equations simultaneously or by maximizing the $\log$-likelihood function given by (27) with respect to $\alpha, \beta, \lambda$ and $\theta$.

\subsection{Method of ordinary and weighted least squares}

(Swain, Venkatraman, and Wilson 1988) proposed the ordinary least squares (OLS) and weighted least squares (WLS) methods to obtain the estimates of the unknown parameters of beta distributions. Here, we consider the two methods to estimate the unknown parameters of the CELP distribution. Let $x_{1: n}<x_{2: n}<\cdots<x_{n: n}$ be the order observations obtained from a sample of size $n$ from the CELP distribution, then the OLS and WLS estimates of $\alpha, \beta, \lambda$ and $\theta$ can be obtained by minimizing the following function with respect to $\alpha, \beta, \lambda$ and $\theta$

$$
S(\alpha, \beta, \theta, \lambda)=\sum_{i=1}^{n} \omega_{i}\left\{\frac{\exp \left[\lambda\left(1-\left(1+\beta x_{i: n}\right)^{-\alpha}\right)^{\theta}\right]-1}{e^{\lambda}-1}-\frac{i}{n+1}\right\}^{2} .
$$

The OLS estimates denoted by $\hat{\alpha}_{O L S}, \hat{\beta}_{O L S}, \hat{\lambda}_{O L S}$ and $\hat{\theta}_{O L S}$ are obtained by setting $\omega_{i}=1$, while we can obtain the WLS estimates denoted by $\hat{\alpha}_{W L S}, \hat{\beta}_{W L S}, \hat{\lambda}_{W L S}$ and $\hat{\theta}_{W L S}$ by setting $\omega_{i}=\frac{(n+1)^{2}(n+2)}{i(n-i+1)}$. These estimates can also be obtained by solving the following equations

$$
\begin{aligned}
& \frac{\partial S(\alpha, \beta, \theta, \lambda)}{\partial \alpha}=\sum_{i=1}^{n} \omega_{i}\left\{\frac{\exp \left[\lambda\left(1-\nu_{i: n}^{-\alpha}\right)^{\theta}\right]-1}{e^{\lambda}-1}-\frac{i}{n+1}\right\} \varphi 1\left(x_{i: n} \mid \alpha, \beta, \theta, \lambda\right), \\
& \frac{\partial S(\alpha, \beta, \theta, \lambda)}{\partial \beta}=\sum_{i=1}^{n} \omega_{i}\left\{\frac{\exp \left[\lambda\left(1-\nu_{i: n}^{-\alpha}\right)^{\theta}\right]-1}{e^{\lambda}-1}-\frac{i}{n+1}\right\} \varphi 2\left(x_{i: n} \mid \alpha, \beta, \theta, \lambda\right), \\
& \frac{\partial S(\alpha, \beta, \theta, \lambda)}{\partial \theta}=\sum_{i=1}^{n} \omega_{i}\left\{\frac{\exp \left[\lambda\left(1-\nu_{i: n}^{-\alpha}\right)^{\theta}\right]-1}{e^{\lambda}-1}-\frac{i}{n+1}\right\} \varphi 3\left(x_{i: n} \mid \alpha, \beta, \theta, \lambda\right)
\end{aligned}
$$


and

$$
\frac{\partial S(\alpha, \beta, \theta, \lambda)}{\partial \lambda}=\sum_{i=1}^{n} \omega_{i}\left\{\frac{\exp \left[\lambda\left(1-\nu_{i: n}^{-\alpha}\right)^{\theta}\right]-1}{e^{\lambda}-1}-\frac{i}{n+1}\right\} \varphi 4\left(x_{i: n} \mid \alpha, \beta, \theta, \lambda\right)
$$

where

$$
\begin{gathered}
\varphi 1\left(x_{i: n} \mid \alpha, \beta, \theta, \lambda\right)=\frac{\lambda \theta \nu_{i: n}^{-\alpha} \log \left(\nu_{i: n}\right)\left(1-\nu_{i: n}^{-\alpha}\right)^{\theta-1} e^{\lambda\left(1-\nu_{i: n}^{-\alpha}\right)^{\theta}}}{e^{\lambda}-1}, \\
\varphi 2\left(x_{i: n} \mid \alpha, \beta, \theta, \lambda\right)=\frac{\lambda \theta \alpha x_{i: n} \nu_{i: n}^{-(\alpha+1)}\left(1-\nu_{i: n}^{-\alpha}\right)^{\theta-1} e^{\lambda\left(1-\nu_{i: n}^{-\alpha}\right)^{\theta}}}{e^{\lambda}-1} \\
\varphi 3\left(x_{i: n} \mid \alpha, \beta, \theta, \lambda\right)=\frac{\lambda\left(1-\nu_{i: n}^{-\alpha}\right)^{\theta} \log \left(1-\nu_{i: n}^{-\alpha}\right) e^{\lambda\left(1-\nu_{i: n}^{-\alpha}\right)^{\theta}}}{e^{\lambda}-1}
\end{gathered}
$$

and

$$
\varphi 4\left(x_{i: n} \mid \alpha, \beta, \theta, \lambda\right)=\frac{\left(e^{\lambda}-1\right) e^{\lambda\left(1-\nu_{i: n}^{-\alpha}\right)^{\theta}}\left(1-\nu_{i: n}^{-\alpha}\right)^{\theta}-e^{\lambda}\left[e^{\lambda\left(1-\nu_{i: n}^{-\alpha}\right)^{\theta}}-1\right]}{\left(e^{\lambda}-1\right)^{2}},
$$

where $\nu_{i: n}, i=1,2, \ldots, n$ are the order observations of $\nu_{i}$ as defined earlier.

\subsection{Method of percentile}

Since the CELP has a closed form distribution function given by (9), then we can use the percentiles (PE) method to estimate the unknown parameters $\alpha, \beta, \lambda$ and $\theta$ by equating the sample percentile points with the corresponding population percentile points, for more details about the PE method see (Kao 1958), (Kao 1959). Let $p_{i}=\frac{i}{n+1}$ be the estimate of $F\left(x_{i: n} \mid \alpha, \beta, \theta, \lambda\right)$, then the PE estimates denoted by $\hat{\alpha}_{P E}, \hat{\beta}_{P E}, \hat{\lambda}_{P E}$ and $\hat{\theta}_{P E}$ can be obtained by minimizing the following function

$$
P(\alpha, \beta, \theta, \lambda)=\sum_{i=1}^{n}\left\{x_{i: n}-\frac{1}{\beta}\left[\left(1-\left(\frac{\log \left[1+p_{i}\left(e^{\lambda}-1\right)\right]}{\lambda}\right)^{1 / \theta}\right)^{-1 / \alpha}-1\right]\right\}^{2}
$$

with respect to $\alpha, \beta, \lambda$ and $\theta$, or equivalently by solving the following non-linear equations

$$
\begin{aligned}
& \frac{\partial P(\alpha, \beta, \theta, \lambda)}{\partial \alpha}=\sum_{i=1}^{n}\left\{x_{i: n}-\frac{1}{\beta}\left[\left(1-\left(v_{i}\right)^{1 / \theta}\right)^{-1 / \alpha}-1\right]\right\} \varpi 1\left(x_{i: n} \mid \alpha, \beta, \theta, \lambda\right), \\
& \frac{\partial P(\alpha, \beta, \theta, \lambda)}{\partial \beta}=\sum_{i=1}^{n}\left\{x_{i: n}-\frac{1}{\beta}\left[\left(1-\left(v_{i}\right)^{1 / \theta}\right)^{-1 / \alpha}-1\right]\right\} \varpi 2\left(x_{i: n} \mid \alpha, \beta, \theta, \lambda\right), \\
& \frac{\partial P(\alpha, \beta, \theta, \lambda)}{\partial \theta}=\sum_{i=1}^{n}\left\{x_{i: n}-\frac{1}{\beta}\left[\left(1-\left(v_{i}\right)^{1 / \theta}\right)^{-1 / \alpha}-1\right]\right\} \varpi 3\left(x_{i: n} \mid \alpha, \beta, \theta, \lambda\right)
\end{aligned}
$$

and

$$
\frac{\partial P(\alpha, \beta, \theta, \lambda)}{\partial \lambda}=\sum_{i=1}^{n}\left\{x_{i: n}-\frac{1}{\beta}\left[\left(1-\left(v_{i}\right)^{1 / \theta}\right)^{-1 / \alpha}-1\right]\right\} \varpi 4\left(x_{i: n} \mid \alpha, \beta, \theta, \lambda\right),
$$

where $v_{i}=\frac{\log \left[1+p_{i}\left(e^{\lambda}-1\right)\right]}{\lambda}$ and

$$
\begin{gathered}
\varpi_{1}\left(x_{i: n} \mid \alpha, \beta, \theta, \lambda\right)=\frac{1}{\beta \alpha^{2}} \log \left(1-v_{i}^{1 / \theta}\right)\left(1-v_{i}^{1 / \theta}\right)^{-1 / \alpha}, \\
\varpi_{2}\left(x_{i: n} \mid \alpha, \beta, \theta, \lambda\right)=\frac{\left[1-\left(v_{i}\right)^{1 / \theta}\right]^{-1 / \alpha}-1}{\beta^{2}},
\end{gathered}
$$




$$
\varpi_{3}\left(x_{i: n} \mid \alpha, \beta, \theta, \lambda\right)=\frac{1}{\beta \alpha \theta^{2}} \log \left(v_{i}\right) v_{i}^{1 / \theta}\left(1-v_{i}^{1 / \theta}\right)^{-1-1 / \alpha}
$$

and

$$
\varpi_{4}\left(x_{i: n} \mid \alpha, \beta, \theta, \lambda\right)=\frac{1}{\beta \alpha \theta \lambda} v_{i}^{1 / \theta-1}\left(1-v_{i}^{1 / \theta}\right)^{-1-1 / \alpha}\left[p_{i} e^{\lambda\left(1-v_{i}\right)}\right] .
$$

\subsection{Method of maximum product of spacing}

According to (Cheng and Amin 1979), (Cheng and Amin 1983), the maximum product of spacing (MPS) estimates of the unknown parameters of the CELP distribution can be obtained based on the idea of differences between the values of the cdf at consecutive data points. Based on a random sample of size $n$ from the CELP distribution, the uniform spacings can be defined as follows

$$
D_{i}(\alpha, \beta, \theta, \lambda)=F\left(x_{i: n} \mid \alpha, \beta, \theta, \lambda\right)-F\left(x_{i-1: n} \mid \alpha, \beta, \theta, \lambda\right), i=1,2, \ldots, n,
$$

where $F(\mid \alpha, \beta, \theta, \lambda)$ is the cdf given by $(9), F\left(x_{0: n} \mid \alpha, \beta, \theta, \lambda\right)=0$ and $F\left(x_{n+1: n} \mid \alpha, \beta, \theta, \lambda\right)=1$. The MPS estimates denoted by $\hat{\alpha}_{M P S}, \hat{\beta}_{M P S}, \hat{\lambda}_{M P S}$ and $\hat{\theta}_{M P S}$ can be obtained by maximizing

$$
\begin{aligned}
M(\alpha, \beta, \theta, \lambda) & =\frac{1}{n+1} \sum_{i=1}^{n+1} \log D_{i}(\alpha, \lambda, a) \\
& =\frac{1}{n+1} \sum_{i=1}^{n+1} \log \left\{\frac{e^{\lambda\left[1-\left(1+\beta x_{i: n}\right)^{-\alpha}\right]^{\theta}}-1}{e^{\lambda}-1}-\frac{e^{\lambda\left[1-\left(1+\beta x_{i-1: n}\right)^{-\alpha}\right]^{\theta}}-1}{e^{\lambda}-1}\right\}
\end{aligned}
$$

with respect to $\alpha, \beta, \lambda$ and $\theta$, or by solving the following equations four equations

$$
\begin{aligned}
& \frac{\partial M(\alpha, \beta, \theta, \lambda)}{\partial \alpha}=\frac{1}{n+1} \sum_{i=1}^{n+1} \frac{\varphi 1\left(x_{i: n} \mid \alpha, \beta, \theta, \lambda\right)-\varphi 1\left(x_{i-1: n} \mid \alpha, \beta, \theta, \lambda\right)}{D_{i}(\alpha, \beta, \theta, \lambda)} \\
& \frac{\partial M(\alpha, \beta, \theta, \lambda)}{\partial \beta}=\frac{1}{n+1} \sum_{i=1}^{n+1} \frac{\varphi\left(x_{i: n} \mid \alpha, \beta, \theta, \lambda\right)-\varphi 2\left(x_{i-1: n} \mid \alpha, \beta, \theta, \lambda\right)}{D_{i}(\alpha, \beta, \theta, \lambda)} \\
& \frac{\partial M(\alpha, \beta, \theta, \lambda)}{\partial \theta}=\frac{1}{n+1} \sum_{i=1}^{n+1} \frac{\varphi 3\left(x_{i: n} \mid \alpha, \beta, \theta, \lambda\right)-\varphi 3\left(x_{i-1: n} \mid \alpha, \beta, \theta, \lambda\right)}{D_{i}(\alpha, \beta, \theta, \lambda)}
\end{aligned}
$$

and

$$
\frac{\partial M(\alpha, \beta, \theta, \lambda)}{\partial \lambda}=\frac{1}{n+1} \sum_{i=1}^{n+1} \frac{\varphi 4\left(x_{i: n} \mid \alpha, \beta, \theta, \lambda\right)-\varphi 4\left(x_{i-1: n} \mid \alpha, \beta, \theta, \lambda\right)}{D_{i}(\alpha, \beta, \theta, \lambda)},
$$

where $\varphi 1\left(x_{i: n} \mid \alpha, \beta, \theta, \lambda\right), \varphi 2\left(x_{i: n} \mid \alpha, \beta, \theta, \lambda\right), \varphi 3\left(x_{i: n} \mid \alpha, \beta, \theta, \lambda\right)$ and $\varphi 4\left(x_{i: n} \mid \alpha, \beta, \theta, \lambda\right)$ are given by (29), (30), (31) and (32).

\subsection{Method of Cramér-von-Mises}

The Cramér-von-Mises estimates (CMEs) denoted by $\hat{\alpha}_{C M E}, \hat{\beta}_{C M E}, \hat{\lambda}_{C M E}$ and $\hat{\theta}_{C M E}$ of $\alpha$, $\beta, \lambda$ and $\theta$ can be obtained by minimizing the following function with respect to $\alpha, \beta, \lambda$ and $\theta$

$$
C(\alpha, \beta, \lambda, \theta)=\frac{1}{12 n}+\sum_{i=1}^{n}\left\{\frac{e^{\lambda\left[1-\left(1+\beta x_{i: n}\right)^{-\alpha}\right]^{\theta}}-1}{e^{\lambda}-1}-\frac{2 i-1}{2 n}-\right\}^{2} .
$$

These estimates also can be obtained by solving the following equations

$$
\frac{\partial C(\alpha, \beta, \lambda, \theta)}{\partial \alpha}=\sum_{i=1}^{n}\left\{\frac{e^{\lambda\left[1-\left(1+\beta x_{i: n}\right)^{-\alpha}\right]^{\theta}}-1}{e^{\lambda}-1}-\frac{2 i-1}{2 n}-\right\} \varphi 1\left(x_{i: n} \mid \alpha, \beta, \lambda, \theta\right),
$$




$$
\begin{aligned}
& \frac{\partial C(\alpha, \beta, \lambda, \theta)}{\partial \beta}=\sum_{i=1}^{n}\left\{\frac{e^{\lambda\left[1-\left(1+\beta x_{i: n}\right)^{-\alpha}\right]^{\theta}}-1}{e^{\lambda}-1}-\frac{2 i-1}{2 n}-\right\} \varphi 2\left(x_{i: n} \mid \alpha, \beta, \lambda, \theta\right), \\
& \frac{\partial C(\alpha, \beta, \lambda, \theta)}{\partial \theta}=\sum_{i=1}^{n}\left\{\frac{e^{\lambda\left[1-\left(1+\beta x_{i: n}\right)^{-\alpha}\right]^{\theta}}-1}{e^{\lambda}-1}-\frac{2 i-1}{2 n}-\right\} \varphi 3\left(x_{i: n} \mid \alpha, \beta, \lambda, \theta\right)
\end{aligned}
$$

and

$$
\frac{\partial C(\alpha, \beta, \lambda, \theta)}{\partial \lambda}=\sum_{i=1}^{n}\left\{\frac{e^{\lambda\left[1-\left(1+\beta x_{i: n}\right)^{-\alpha}\right]^{\theta}}-1}{e^{\lambda}-1}-\frac{2 i-1}{2 n}-\right\} \varphi 3\left(x_{i: n} \mid \alpha, \beta, \lambda, \theta\right),
$$

where $\varphi 1\left(x_{i: n} \mid \alpha, \beta, \theta, \lambda\right), \varphi 2\left(x_{i: n} \mid \alpha, \beta, \theta, \lambda\right), \varphi 3\left(x_{i: n} \mid \alpha, \beta, \theta, \lambda\right)$ and $\varphi 4\left(x_{i: n} \mid \alpha, \beta, \theta, \lambda\right)$ are defined by (29), (30), (31) and (32).

\section{Simulation study}

It is not possible to compare the performance of the differernt estimators derived in the previous sections theoretically, therfore, we conduct a Monte Carlo simulation study to determine the best estimation method that can be used to estimate the CELP distribution paramteres. We generate 1000 random samples of sizes 50,100 and 200 from CELP distrribution and by considering $\lambda=1$ in all setting and by choosing $\alpha=(0.5,1.5), \beta=(0.5,1)$ and $\theta=(1,2)$. In each comination from eight parameter combinations, we obtain the average value of estimates as well as the root mean square error (RMSE) as follows

$$
\operatorname{Mean}\left(\hat{\mu}_{j}\right)=\frac{1}{1000} \sum_{i=1}^{1000} \hat{\mu}_{j, i} \text { and } \operatorname{RMSE}\left(\hat{\mu}_{j}\right)=\sqrt{\frac{1}{1000} \sum_{i=1}^{1000}\left(\hat{\mu}_{j, i}-\mu_{j}\right)^{2}}
$$

where $\boldsymbol{\mu}=(\alpha, \beta, \lambda, \theta)$ and $\hat{\boldsymbol{\mu}}=(\hat{\alpha}, \hat{\beta}, \hat{\lambda}, \hat{\theta})$. All the estimators are obtained by solving four nonlinear equations. This is a very popular problem in the literature and some numerical techniques can be used to obtain the estimates. We first estimated the parameters using the method of maximum likelihood by maximizing the log-likelihood function using the Quasi Newton method. For all other methods, the maximum likelihood estimates were used as the initial values. These values are displayed for the different six methods of estimation in Tables 2-4. The simulation study is carried out using Mathcad program version 2007. From these Tables, we observe that the RMSE decreases as the sample size increases, which means that the different methods provide a consistent estimators. The PCEs perform better than other estimates in terms of minimum RMSE in most cases followed by WLSEs, LSEs, CMEs, MPSEs and MLEs. 
Table 2: Average values of estimates and the corresponding RMSEs (in parentheses) for $n=50$

\begin{tabular}{|c|c|c|c|c|c|c|}
\hline 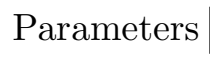 & MLEs & LSEs & WLSEs & PCEs & MPSEs & CMEs \\
\hline & ) & & & & & \\
\hline & & $0.548(0.352)$ & $0.504(0.332)$ & $460(0.258)$ & 0.707 & 0.757 \\
\hline & & $1.063(0.399)$ & $1.088(0.423)$ & & 0.991 & $0.790(0.39$ \\
\hline & 3) & $0.746(0$ & 0.530 & $1.186(0$ & 1.188 & 0.956 \\
\hline , & 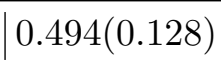 & ) & ) & $0.547(0.211)$ & 56 & $5:$ \\
\hline & 32 & $0.435(($ & 0.460 & 486 & 0.68 & 0.45 \\
\hline & & $1.829(0$ & 1.796 & $2.757(1$ & 2.146 & \\
\hline & & $0.692(1$ & 0.619 & $1.160(0$. & 0.834 & 0.977 \\
\hline & ) & ) & & $1261(0,00)$ & & \\
\hline & & 0.4 & 0.5 & & 0.3 & \\
\hline & & 1.018 & 0.960 & 0.725 & 0.81 & 1.06 \\
\hline & 868 & 1.003 & 1.18 & 1.016 & 1.32 & \\
\hline & 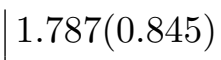 & 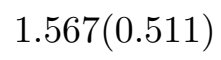 & & & & \\
\hline & & 0 & 0 & & & \\
\hline & & & 2.2 & & & \\
\hline & 0.543 & 0.619 & 0.615 & 0.963 & 1.1 & 0.38 \\
\hline$\alpha=0$. & ner & ( & 0.522 & 522 & 0.6 & 0 . \\
\hline & & & & & & \\
\hline & & 0.9 & 0.9 & & 0.8 & \\
\hline$\lambda=1$ & 0.455 & 0.685 & 0.72 & 1.094 & 1.23 & 0.80 \\
\hline & & & & & & \\
\hline & & & & & & \\
\hline & ) & 1.714 & 1.7 & 2) & 1.8 & 1.78 \\
\hline$\lambda=1$ & 1.342( & $0.855(1$ & 0.769 & $0.961(0$ & 0.808 & $0.876(1.323)$ \\
\hline & & & & & & \\
\hline & ) & 2.1 & 2.1 & P 270 & & 2.3 \\
\hline & ) & 1.104 & 1.0 & 0.840 & 0.91 & 1.19 \\
\hline$\lambda=1$ & 0.680 & $0.875(0$ & 0.975 & 1.074( & 0.93 & 0.927 \\
\hline & & 1.0 & Lar & & 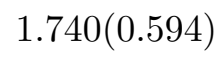 & 20 \\
\hline & & & 1.77 & & 2.5 & 2.53 \\
\hline & $2.723(2.141)$ & $1.967(0$ & $1.930(0.8$ & $1.462(1.1$ & $2.295(2.2$ & $2.254(1.192)$ \\
\hline & $0.606(1.682)$ & $0.678(1.283)$ & $0.691(1.473)$ & $1.129(0.431)$ & $0.972(1.775)$ & $1.049(1.393)$ \\
\hline
\end{tabular}


Table 3: Average values of estimates and the corresponding RMSEs (in parentheses) for $n=100$

\begin{tabular}{|c|c|c|c|c|c|c|}
\hline Parar & MLEs & LSEs & WLSEs & PCEs & MPSEs & CMEs \\
\hline$\alpha=0.5$ & $0.467(0$. & $476(0.082)$ & $74(0.078)$ & $0.504(0.177)$ & 04) & 1) \\
\hline$\beta=0.5$ & $0.755(0.722)$ & $0.526(0.316)$ & $0.526(0.297)$ & $0.499(0.253)$ & $0.692(0.819)$ & $0.644(0.990)$ \\
\hline$\theta=1$ & $1.143(0.547)$ & $0.966(0.294)$ & $0.996(0.280)$ & $1.393(0.800)$ & $0.946(0.478)$ & $0.905(0.327)$ \\
\hline$\lambda=1$ & $0.726(1.697)$ & $0.799(0.851)$ & $0.737(0.954)$ & $1.179(0.353)$ & $1.187(1.936)$ & $0.958(1.602)$ \\
\hline$\alpha=$ & $0.473(0.096)$ & $0.482(0.087)$ & $0.479(0.084)$ & $0.509(0.158)$ & $0.503(0.090)$ & $0.498(0.074)$ \\
\hline$\beta=0.5$ & $0.434(0.282)$ & $0.485(0.302)$ & $0.477(0.300)$ & $0.388(0.220)$ & $0.580(0.617)$ & $0.472(0.258)$ \\
\hline$\theta=2$ & $1.895(0.768)$ & $1.690(0.597)$ & $1.819(0.637)$ & $2.562(1.436)$ & $1.956(1.119)$ & $1.753(0.585)$ \\
\hline$\lambda=1$ & $0.860(1.844)$ & $0.991(1.127)$ & $0.835(1.152)$ & $1.126(0.307)$ & $0.879(1.331)$ & $1.073(0.881)$ \\
\hline$\alpha=1.5$ & $870(0.833)$ & $1.623(0.443)$ & $1.736(0.652)$ & $1.464(0.660)$ & 2.160 & 1.772 \\
\hline$\beta=0.5$ & $0.487(0.385)$ & $0.475(0.251)$ & $0.474(0.276)$ & $0.586(0.289)$ & 0.364( & $0.484(0.343)$ \\
\hline$\theta$ & $0.955(0.341)$ & $0.995(0.314)$ & $0.930(0.290)$ & $0.820(0.556)$ & $0.825(0.339)$ & $1.009(0.368)$ \\
\hline$\lambda=1$ & $0.990(1.565)$ & $0.947(0.513)$ & $1.119(1.098)$ & $1.087(0.250)$ & $1.222(1.447)$ & $0.981(0.754)$ \\
\hline$\alpha=1.5$ & $604(0.472)$ & $1.570(0.318)$ & $1.592(0.415)$ & $1.362(0.545)$ & $1.750(0.482)$ & 1.71 \\
\hline & $0.568(0.533)$ & $0.455(0.206)$ & $0.503(c$ & $0.581(0.1$ & 0.398 & 0.467 \\
\hline$\theta=2$ & $2.327(1.690)$ & $1.991(0.645)$ & $1.975(0.785)$ & $1.719(1.099)$ & $1.765(0.908)$ & $2.091(0.942)$ \\
\hline$\lambda=1$ & $0.638(1.341)$ & $0.785(0.585)$ & $1.013(1.126)$ & $1.096(0.242)$ & $0.923(1.490)$ & $0.786(0.627)$ \\
\hline$\alpha=0.5$ & 486 & $0.512(0.103)$ & 0.511 ( & $0.525(0.200)$ & $0.537(0$ & 0.520 \\
\hline & $0.978(0.837)$ & $0.822(0.565)$ & $0.903(0.577)$ & $0.839(0.4$ & $0.975(1.1$ & $0.938(0.595)$ \\
\hline$\theta=1$ & $1.002(0.382)$ & $0.930(0.364)$ & $0.969(0.3$ & $1.203(0.6$ & 0.895( & $0.966(0.435)$ \\
\hline$\lambda=1$ & $0.697(1.351)$ & $0.782(0.752)$ & $0.821(0.628)$ & $1.119(0.206)$ & $0.883(1.736)$ & $0.989(1.096)$ \\
\hline$\alpha=0.5$ & (10- & $0.487(0.0$ & 0.491( & $0.488(0$. & 0.516 & 0.507 \\
\hline & $1.266(0.795)$ & $1.004(0.466)$ & $1.018(0.436)$ & $1.049(0.513)$ & $1.078(1.067)$ & $1.103(0.643)$ \\
\hline$\theta=2$ & $2.148(0.805)$ & $1.880(0.705)$ & $1.925(0.734)$ & $2.058(1.056)$ & $1.880(1.077)$ & $1.948(0.704)$ \\
\hline$\lambda=1$ & $0.973(1.052)$ & $1.060(1.162)$ & $1.061(1.232)$ & $0.975(0.234)$ & $0.993(1.262)$ & $0.990(0.829)$ \\
\hline & 1.09 & & $1.4^{\prime}$ & 1.40 & 1.56 & \\
\hline & $2.080(1.232)$ & $2.151(0.5$ & 2.045( & $1.977(0$. & $2.076(1.1 \%$ & $2.312(1.053)$ \\
\hline & $1.040(0.335)$ & $1.052(0.330)$ & $1.042(0.297)$ & $0.940(0.557)$ & $0.945(0.286)$ & $1.095(0.361)$ \\
\hline$\lambda=1$ & $0.849(1.727)$ & $0.940(0.893)$ & $0.909(0.862)$ & $0.906(0.216)$ & $1.004(0.989)$ & $1.019(0.771)$ \\
\hline & 1.526 & 1.48 & 1.48 & 1.40 & 1.571 & 1.50 \\
\hline$\beta=2$ & $2.150(1.056)$ & $2.056(0.630)$ & $2.052(0.652)$ & $2.005(0.599)$ & $1.946(1.180)$ & $2.240(1.020)$ \\
\hline & $2.202(0.819)$ & $2.026(0.698)$ & $2.048(0.636)$ & $1.680(0.941)$ & $1.932(0.795)$ & $2.060(0.753)$ \\
\hline$\lambda=1$ & $0.665(1.503)$ & $0.766(1.010)$ & $0.768(1.084)$ & $0.950(0.155)$ & $0.962(1.268)$ & $1.032(1.112)$ \\
\hline
\end{tabular}


Table 4: Average values of estimates and the corresponding RMSEs (in parentheses) for $n=200$

\begin{tabular}{|c|c|c|c|c|c|c|}
\hline Parameters & MLEs & LSEs & WLSEs & PCEs & MPSEs & CMEs \\
\hline$\alpha=0.5$ & $0.489(0.073)$ & $0.501(0.062)$ & $0.489(0.061)$ & $0.503(0.165)$ & $0.502(0.070)$ & $0.509(0.085)$ \\
\hline$\beta=0.5$ & $0.637(0.392)$ & $0.512(0.304)$ & $.521(0.280)$ & $0.504(0.221)$ & $0.570(0.470)$ & $0.634(0.353)$ \\
\hline$\theta=1$ & $1.047(0.328)$ & $0.989(0.196)$ & $1.002(0.224)$ & $1.387(0.524)$ & $0.939(0.341)$ & $1.052(0.462)$ \\
\hline$\lambda=1$ & $1.117(1.670)$ & $0.973(0.634)$ & $0.941(0.807)$ & $1.174(0.351)$ & $1.157(1.849)$ & $1.151(0.989)$ \\
\hline$\alpha=0.5$ & $0.485(0.072)$ & $0.493(0.063)$ & $0.488(0.064)$ & $0.509(0.156)$ & $0.499(0.062)$ & $0.497(0.055)$ \\
\hline$\beta=0.5$ & $0.515(0.224)$ & $0.474(0.280)$ & $0.508(0.277)$ & $0.401(0.258)$ & $0.470(0.302)$ & $0.455(0.253)$ \\
\hline$\theta=2$ & $2.040(0.680)$ & $1.705(0.542)$ & $1.897(0.497)$ & $2.329(1.172)$ & $1.857(0.689)$ & $1.769(0.548)$ \\
\hline$\lambda=1$ & $0.937(1.826)$ & $1.053(0.940)$ & $0.882(1.005)$ & $1.065(0.264)$ & $0.932(1.164)$ & $0.970(0.736)$ \\
\hline$\alpha=$ & 41) & ) & 3) & ) & 1.7 & 1.0 \\
\hline$\beta=0.5$ & $0.539(0$. & $0.563(($ & 0.557( & 0.538 & 0.41 & 0.574 \\
\hline$\theta=1$ & $1.033(0.238)$ & $1.061(0.253)$ & $1.022(0.241)$ & $0.835(0$ & 0.957 & $1.057(0.284)$ \\
\hline$\lambda=1$ & $0.981(1.145)$ & $0.946(0.484)$ & $1.032(0.890)$ & $1.087(0.219)$ & $0.910(1.127)$ & $0.993(0.6$ \\
\hline$\alpha=1.5$ & $1.569(0.266)$ & $1.568(0.225)$ & $1.599(0.291)$ & $1.368(0.469)$ & $1.606(0.268)$ & $1.653(0.433)$ \\
\hline & 0.466 & 0.455 & 0.46 & 0.599 & $0.455(c$ & 0.471( \\
\hline$\theta=2$ & $1.934(0.474)$ & $1.944(0$ & & & 1.711 & 1.988 \\
\hline$\lambda=1$ & $0.779(1.179)$ & $0.891(0.492)$ & $0.949(0.754)$ & $1.102(0.229)$ & $1.073(1.376)$ & $0.909(0.574)$ \\
\hline$\alpha=0.5$ & 47 & 0.502 & 0.50 & 0.51 & 0.5 & 0.50 \\
\hline & $0.907(0.4$ & 1.060 & 1.019 & 0.89 & 1.01 & 1.152 \\
\hline$\theta=1$ & $0.994(0.287)$ & $1.042(0.333)$ & $1.029(0.252)$ & $1.098(0.529)$ & 0.921 & $1.036(0.185)$ \\
\hline$\lambda=1$ & $0.907(0.902)$ & $0.904(0.679)$ & $0.905(0.568)$ & $1.036(0.154)$ & $1.082(1.152)$ & $0.956(0.563)$ \\
\hline & & & & & 0.5 & \\
\hline & $1.124(0.661)$ & $1.000(0.447)$ & & 0.980 & 1.012 & $0.970(0.436)$ \\
\hline & $2.025(0.781)$ & $1.921(0.657)$ & $1.993(0.529)$ & $2.022(0.924)$ & $1.927(0.804)$ & $1.984(0.446)$ \\
\hline$\lambda=1$ & $1.069(0.914)$ & $1.007(0.867)$ & $1.050(0.904)$ & $1.036(0.231)$ & $0.928(0.708)$ & $0.921(0.532)$ \\
\hline$\alpha=1.5$ & $1.553(0.330)$ & $1.444(0$ & 1.46 & 1.433 & 1.51 & 1.512 \\
\hline & $2.028(0.938)$ & $2.085(0.360)$ & $2.003(0.270)$ & $1.996(0.625)$ & $1.964(0.825)$ & $2.129(0.699)$ \\
\hline & $0.957(0.234)$ & $0.998(0.240)$ & $1.004(0.210)$ & $0.910(0.512)$ & $0.941(0.195)$ & $1.023(0.237)$ \\
\hline$\lambda=1$ & $1.079(1.332)$ & $1.003(0.660)$ & $0.925(0.673)$ & $1.020(0.201)$ & $0.943(0.602)$ & $1.007(0.445)$ \\
\hline$\alpha=1.5$ & $1.541(0.272)$ & $1.520(0.201)$ & $1.519(0.200)$ & $1.481(0.40$ & 1.539 & $1.500(0$ \\
\hline & $1.993(0.971)$ & $1.977(0.508)$ & $2.032(0.636)$ & $2.019(0.570)$ & $1.957(0.914)$ & $1.980(0.648)$ \\
\hline & $1.904(0.504)$ & $1.935(0.409)$ & $1.998(0.531)$ & $1.703(0.851)$ & $1.994(0.494)$ & $1.954(0.531)$ \\
\hline$\lambda=1$ & $1.046(1.458)$ & $1.065(0.521)$ & $1.040(0.524)$ & $1.007(0.112)$ & $1.003(0.722)$ & $1.016(0.699)$ \\
\hline
\end{tabular}

\section{Applications}

In this section, we provide two applications to real data sets to illustrate the importance of the CELP distribution presented in Section 2. The MLEs of the model parameters are computed and goodness-of-fit statistics for these models are compared with other competing models. The first real data set was originally reported by (Lee and Wang 2003), which represents the remission times (in months) of a random sample of 128 bladder cancer patients. The cancer data were analyzed by (Mead and Afify 2017) and (Aldahlan and Afify 2018). The Second data set was taken from (Murthy, Xie, and Jiang 2004), which represents the failures times of 50 items.

We compare the fits of the new CELP distribution with some competative models which are listed in Table 5 and their densities are given by: 
$\mathrm{TL}: f(x)=\alpha \beta(1+\beta x)^{-\alpha-1}\left[1-\lambda+2 \lambda(1+\beta x)^{-\alpha}\right]$

BEL: $f(x)=\frac{\beta \theta \lambda}{B(a, b)}(1+\lambda x)^{-\theta-1}\left[1-(1+\lambda x)^{-\theta}\right]^{a \beta-1}\left\{1-\left[1-(1+\lambda x)^{-\theta}\right]^{\beta}\right\}^{b-1}$;

McL: $f(x)=\frac{c \alpha \beta^{\alpha}}{B(a / c, \eta+1)}(\beta+x)^{-\alpha-1}\left[1-\left(\frac{\beta}{\beta+x}\right)^{\alpha}\right]^{a-1}\left\{1-\left[1-\left(\frac{\beta}{\beta+x}\right)^{\alpha}\right]^{c}\right\}^{\eta}$

$\mathrm{WL}: f(x)=\frac{a b \alpha}{\beta}\left(1+\frac{x}{\beta}\right)^{b \alpha-1}\left[1-\left(1+\frac{x}{\beta}\right)^{-\alpha}\right]^{b-1} \mathrm{e}^{-a\left[\left(1+\frac{x}{\beta}\right)^{\alpha}-1\right]^{b}}$

BXL: $f(x)=\frac{2 \theta \alpha}{\beta}\left(1+\frac{x}{\beta}\right)^{\alpha-1}\left[\left(1+\frac{x}{\beta}\right)^{\alpha}-1\right] \mathrm{e}^{-\left[\left(1+\frac{x}{\beta}\right)^{\alpha}-1\right]^{2}}\left\{1-\mathrm{e}^{-\left[\left(1+\frac{x}{\beta}\right)^{\alpha}-1\right]^{2}}\right\}^{\theta-1}$.

The parameters of the above densities are positive real numbers, except $|\lambda| \leq 1$ for the TL model.

The fitted models are compared using goodness-of-fit measures, namely: the maximized loglikelihood under the model $(-\widehat{\ell})$, Cramér-Von Mises (CVM), Anderson-Darling (AD), Kolmogorov Smirnov (KS) statistic and its p-value (PV). The values of goodness-of-fit statistics, the MLEs and their corresponding standard errors (SEs) (listed in parentheses) of the fitted models are given in Tables 6 and 7, respectively. It is clear that the CELP distribution fits very well for both the data sets. Figures 3 and 4 show the fitted pdf, cdf, sf and PP plots of the CELP distribution for both the data sets, respectively.

Table 5: The fitted competitive models

\begin{tabular}{ll}
\hline Distribution & Author(s) \\
\hline Transmuted Lomax (TL) & (Ashour and Eltehiwy 2013) \\
Beta exponentiated Lomax (BEL) & (Mead 2016) \\
McDonald Lomax (McL) & (Lemonte and Cordeiro 2013) \\
Exponentiated Lomax (EL) & (Abdul-Moniem and Abdel-Hameed 2012) \\
Weibull Lomax (WL) & (Tahir et al. 2015) \\
Burr X Lomax (BXL) & (Yousof et al. 2017$)$ \\
Lomax (L) & (Lomax 1954) \\
\hline
\end{tabular}


Table 6: Goodness-of-fit statistics, MLEs and SEs for cancer data

\begin{tabular}{|c|c|c|c|c|c|c|}
\hline Model & $-\widehat{\ell}$ & CVM & $\mathrm{AD}$ & $\mathrm{KS}$ & $\mathrm{PV}$ & Estimates (SEs) \\
\hline \multirow[t]{4}{*}{ CELP } & 409.385 & 0.013 & 0.084 & 0.028 & 0.999 & $\begin{array}{ll}\alpha & 2.872(0.888)\end{array}$ \\
\hline & & & & & & $\beta \quad 0.121(0.072)$ \\
\hline & & & & & & $\begin{array}{ll}\theta & 1.027(0.707)\end{array}$ \\
\hline & & & & & & $\lambda \quad 3.263(2.444)$ \\
\hline \multirow[t]{3}{*}{$\mathrm{TL}$} & 409.625 & 0.019 & 0.125 & 0.035 & 0.996 & $\alpha \quad 4.016(1.486)$ \\
\hline & & & & & & $\beta \quad 0.052(0.025)$ \\
\hline & & & & & & $\begin{array}{ll}\lambda & -0.844(0.139)\end{array}$ \\
\hline \multirow[t]{5}{*}{ BEL } & 409.927 & 0.025 & 0.168 & 0.039 & 0.989 & $\beta \quad 2.693(4.883)$ \\
\hline & & & & & & $\theta \quad 1.446(4.180)$ \\
\hline & & & & & & $\lambda \quad 0.077(0.112)$ \\
\hline & & & & & & $a \quad 0.565(0.986)$ \\
\hline & & & & & & $b \quad 2.703(8.117)$ \\
\hline \multirow[t]{5}{*}{ McL } & 409.912 & 0.025 & 0.168 & 0.039 & 0.989 & $\alpha \quad 0.808(3.545)$ \\
\hline & & & & & & $\beta \quad 11.29(18.29)$ \\
\hline & & & & & & $a \quad 1.506(0.283)$ \\
\hline & & & & & & $b \quad 4.188(26.36)$ \\
\hline & & & & & & c $2.104(3.111)$ \\
\hline \multirow[t]{3}{*}{ EL } & 410.071 & 0.028 & 0.189 & 0.040 & 0.984 & $\alpha \quad 4.570(2.117)$ \\
\hline & & & & & & $\beta \quad 0.040(0.026)$ \\
\hline & & & & & & $\theta \quad 1.587(0.274)$ \\
\hline \multirow[t]{4}{*}{ WL } & 410.079 & 0.030 & 0.201 & 0.041 & 0.978 & $\alpha \quad 0.110(0.173)$ \\
\hline & & & & & & $\beta \quad 6.881(6.791)$ \\
\hline & & & & & & $a \quad 35.52(103.4)$ \\
\hline & & & & & & $b \quad 1.518(0.287)$ \\
\hline \multirow[t]{3}{*}{ BXL } & 411.145 & 0.054 & 0.354 & 0.047 & 0.932 & $\alpha \quad 0.298(0.051)$ \\
\hline & & & & & & $\beta \quad 1.020(0.664)$ \\
\hline & & & & & & $\theta \quad 0.933(0.249)$ \\
\hline \multirow[t]{2}{*}{$\mathrm{L}$} & 413.836 & 0.077 & 0.471 & 0.097 & 0.174 & $\alpha \quad 12.77(5.964)$ \\
\hline & & & & & & $\beta \quad 0.009(0.004)$ \\
\hline
\end{tabular}



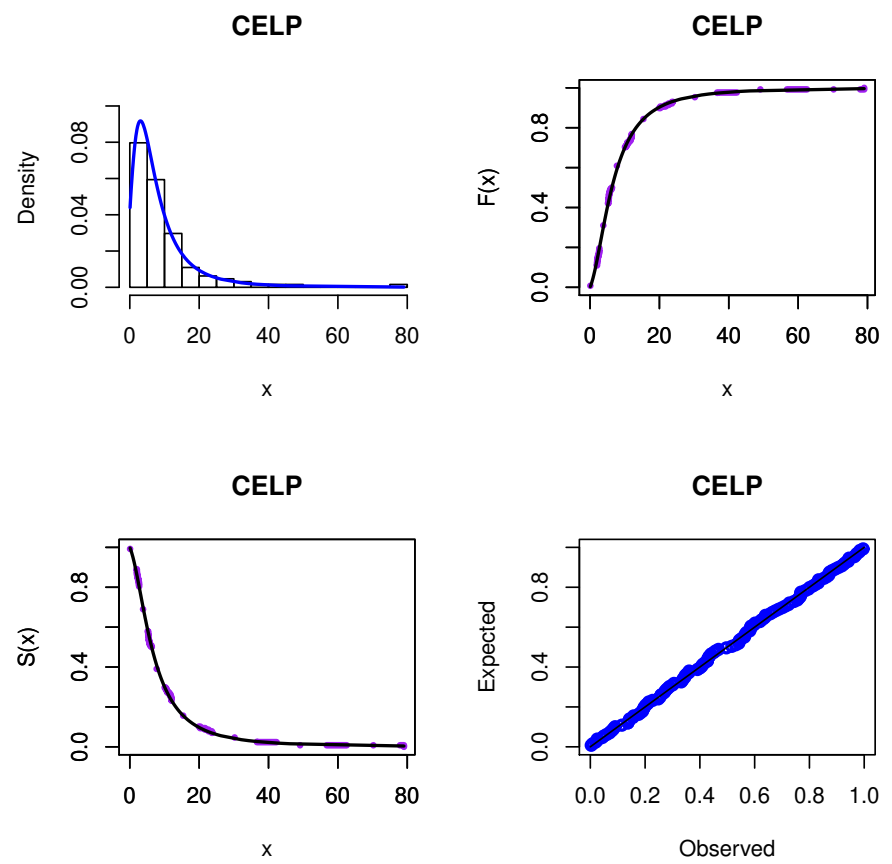

Figure 3: Fitted pdf, cdf, sf and PP plots for cancer data
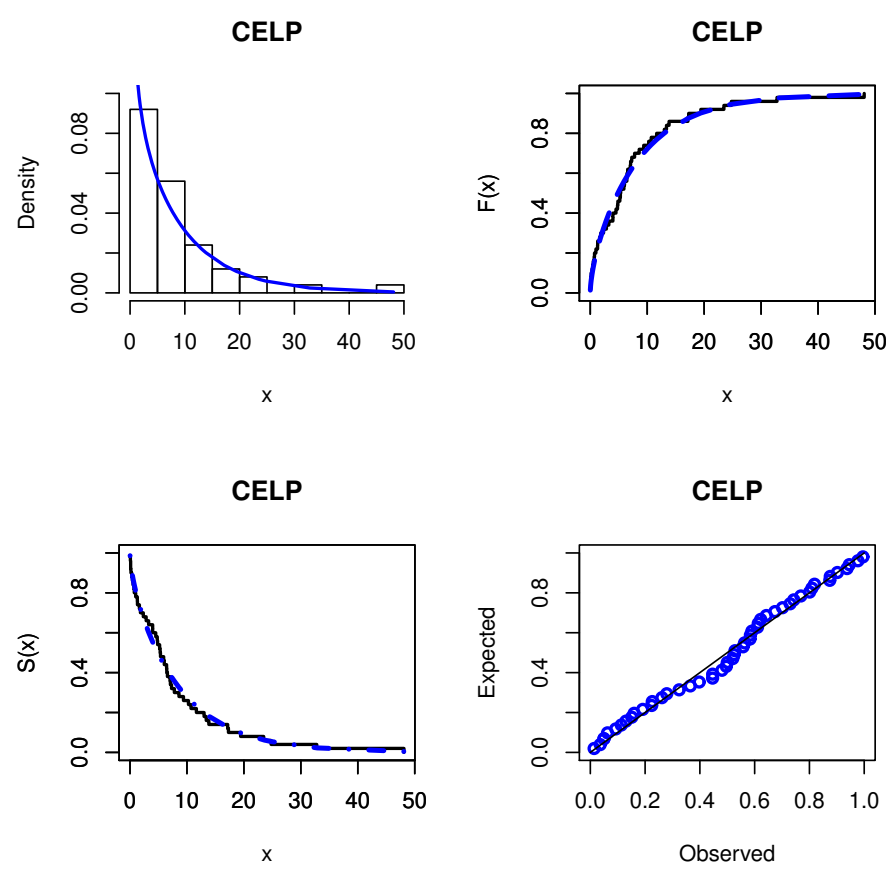

Figure 4: Fitted pdf, cdf, sf and PP plots for failures times data 
Table 7: Goodness-of-fit statistics, MLEs and SEs for failures times data

\begin{tabular}{|c|c|c|c|c|c|c|c|}
\hline Model & $-\widehat{\ell}$ & CVM & $\mathrm{AD}$ & KS & $\mathrm{PV}$ & \multicolumn{2}{|c|}{ Estimates (SEs) } \\
\hline \multirow[t]{4}{*}{ CELP } & 150.152 & 0.050 & 0.261 & 0.086 & 0.850 & $\alpha$ & $52.72(1.498)$ \\
\hline & & & & & & $\beta$ & $0.002(0.0003)$ \\
\hline & & & & & & $\theta$ & $0.587(0.229)$ \\
\hline & & & & & & $\lambda$ & $0.875(1.533)$ \\
\hline \multirow[t]{3}{*}{ TL } & 152.150 & 0.088 & 0.444 & 0.094 & 0.763 & $\alpha$ & $23.41(14.01)$ \\
\hline & & & & & & $\beta$ & $0.004(0.001)$ \\
\hline & & & & & & $\lambda$ & $0.467(0.654)$ \\
\hline \multirow[t]{5}{*}{ BEL } & 150.145 & 0.055 & 0.279 & 0.094 & 0.766 & $\beta$ & $2.246(4.229)$ \\
\hline & & & & & & $\theta$ & $3.426(14.73)$ \\
\hline & & & & & & $\lambda$ & $0.011(0.016)$ \\
\hline & & & & & & $a$ & $0.289(0.530)$ \\
\hline & & & & & & $b$ & $3.862(17.46)$ \\
\hline \multirow[t]{5}{*}{$\mathrm{McL}$} & 150.131 & 0.054 & 0.276 & 0.094 & 0.768 & $\alpha$ & $0.386(13.41)$ \\
\hline & & & & & & $\beta$ & $31.84(223)$ \\
\hline & & & & & & $a$ & $0.648(0.136)$ \\
\hline & & & & & & $b$ & $36.22(1864)$ \\
\hline & & & & & & $c$ & $2.068(4.780)$ \\
\hline \multirow[t]{3}{*}{ EL } & 150.343 & 0.069 & 0.344 & 0.107 & 0.612 & $\alpha$ & $23.43(11.65)$ \\
\hline & & & & & & $\beta$ & $0.004(0.002)$ \\
\hline & & & & & & $\theta$ & $0.700(0.122)$ \\
\hline \multirow[t]{4}{*}{ WL } & 150.681 & 0.085 & 0.428 & 0.111 & 0.558 & $\alpha$ & $0.012(0.020)$ \\
\hline & & & & & & $\beta$ & $6032(76.56)$ \\
\hline & & & & & & $a$ & $7407(12905)$ \\
\hline & & & & & & $b$ & $0.800(0.095)$ \\
\hline \multirow[t]{3}{*}{ BXL } & 150.327 & 0.062 & 0.316 & 0.097 & 0.726 & $\alpha$ & $0.482(0.169)$ \\
\hline & & & & & & $\beta$ & $5.134(4.095)$ \\
\hline & & & & & & $\theta$ & $0.334(0.075)$ \\
\hline \multirow[t]{2}{*}{$\mathrm{L}$} & 152.573 & 0.069 & 0.349 & 0.105 & 0.638 & $\alpha$ & $28.56(13.17)$ \\
\hline & & & & & & $\beta$ & $0.004(0.002)$ \\
\hline
\end{tabular}



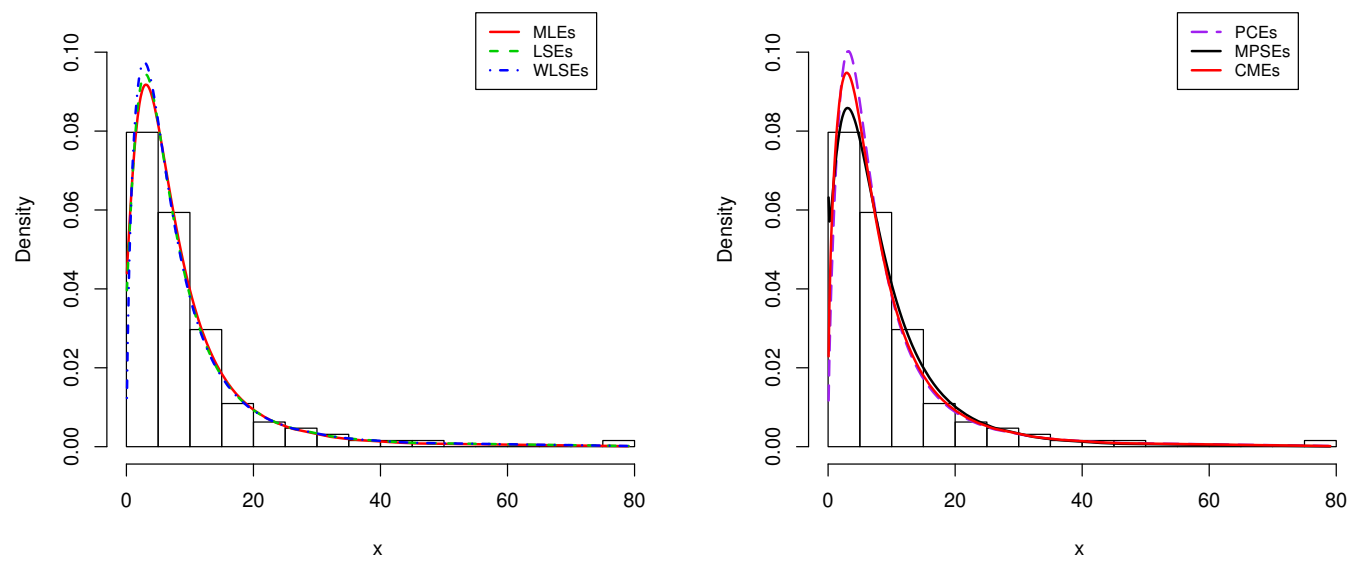

Figure 5: The fitted CELP PDF for different estimation methods for cancer data
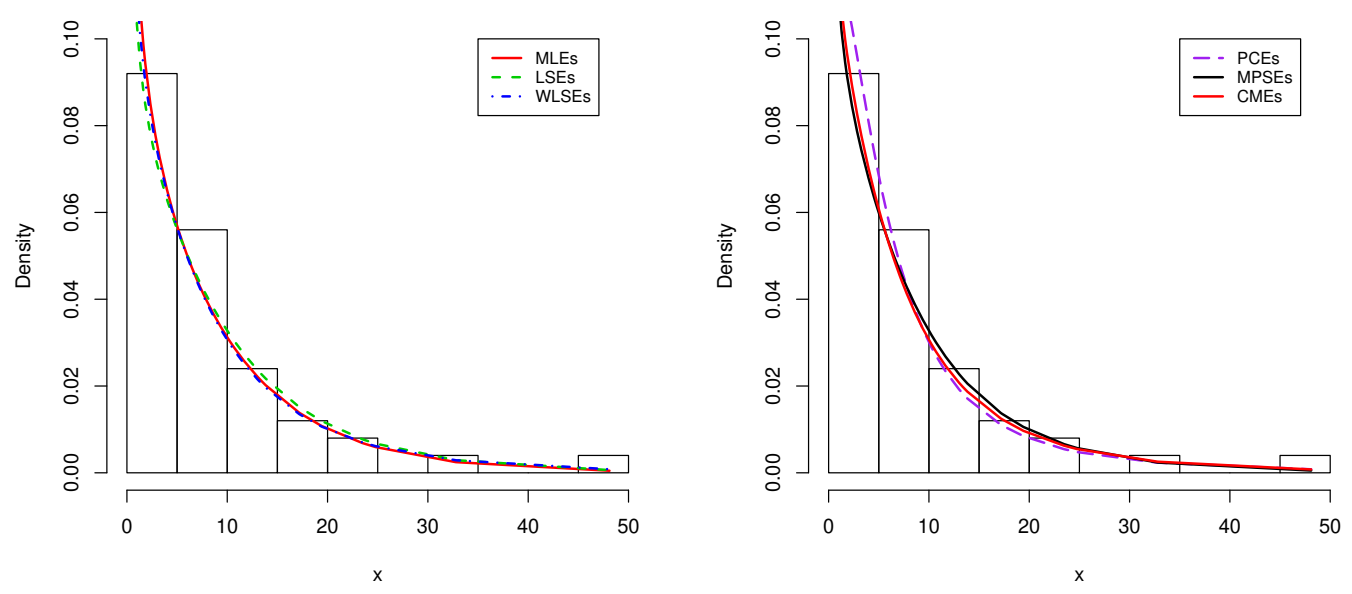

Figure 6: The fitted CELP PDF for different estimation methods failures times data

We obtain the estimates of the unknown parameters of the CELP distribution using six methods of estimation and the values of $-\widehat{\ell}, \mathrm{KS}$ and corresponding $\mathrm{P}$-values are displayed in Tables 8 and 9 for both the data sets. The values in Tables 8 and 9 reveal that the MLE method can be used to estimate the parameters of the CELP distribution for bladder cancer data, whereas, the LSE method can be used to estimate the parameters of the CELP distribution for failures times data. However, all estimation methods perform well. The histogram of the data and the fitted densities for estimation methods are displayed in Figures 5 and 6 . These Figures support the results in Tables 8 and 9.

\section{Conclusion}

In this article, we have proposed a new distribution, called the complementary exponentiated Lomax-Poisson distribution and discussed its various properties. We have observed that 
Table 8: The parameters estimates of the CELP model using different methods, $-\widehat{\ell}, \mathrm{KS}$ statistic and corresponding PV for bladder cancer data

\begin{tabular}{lccccccc}
\hline Method & $\alpha$ & $\beta$ & $\theta$ & $\lambda$ & $-\widehat{\ell}$ & KS & PV \\
\hline MLEs & 2.872 & 0.121 & 1.027 & 3.263 & 409.385 & 0.028 & 0.9999 \\
LSEs & 2.454 & 0.162 & 1.022 & 3.626 & 409.536 & 0.031 & 0.9997 \\
WLSEs & 2.349 & 0.186 & 1.909 & 1.898 & 409.509 & 0.033 & 0.9989 \\
PCEs & 2.204 & 0.255 & 1.806 & 3.043 & 410.814 & 0.035 & 0.9977 \\
MPSEs & 3.896 & 0.065 & 0.559 & 4.540 & 409.920 & 0.039 & 0.9894 \\
CMEs & 2.540 & 0.159 & 1.536 & 2.377 & 409.540 & 0.031 & 0.9997 \\
\hline
\end{tabular}

Table 9: The parameters estimates of the CELP model using different methods, $-\widehat{\ell}, \mathrm{KS}$ statistic and corresponding PV for failures times data

\begin{tabular}{lccccccc}
\hline Method & $\alpha$ & $\beta$ & $\theta$ & $\lambda$ & $-\widehat{\ell}$ & KS & PV \\
\hline MLEs & 52.72 & 0.002 & 0.587 & 0.875 & 150.152 & 0.086 & 0.8501 \\
LSEs & 23.20 & 0.005 & 0.326 & 2.892 & 150.544 & 0.077 & 0.9298 \\
WLSEs & 6.074 & 0.022 & 0.343 & 2.794 & 150.487 & 0.084 & 0.8732 \\
PCEs & 2.406 & 0.118 & 0.601 & 2.938 & 152.062 & 0.094 & 0.7709 \\
MPSEs & 10.72 & 0.013 & 0.425 & 2.341 & 150.484 & 0.085 & 0.8607 \\
CMEs & 4.097 & 0.041 & 0.378 & 3.001 & 150.473 & 0.079 & 0.9105 \\
\hline
\end{tabular}

the proposed distribution is more flexible than some of the existing models. We have also observed that it has decreasing, increasing and unimodal hazard rate depending upon the parameters. The estimation of model parameters are outlined using six different frequentist methods of estimation. Two applications to real data sets are presented as an illustration of the potentiality of the new model as compared to some existing models. After comparing the values of four popular goodness-of-fit statistics, we may say that our model CELP distribution is better as compared to others for these two data sets. We expect the utility of the newly proposed model in different fields especially in reliability when the hazard rate is decreasing, increasing or unimodal (upside-down bathtub). The new model is analytically quite tractable, and it can be used quite effectively, to analyze censored data also.

\section{Acknowledgments}

The authors would like to thank the Editor, Associate Editor and the referees for careful reading and for valuable comments that greatly improved the presentation of article.

\section{References}

Abd-Elfattah AM, Alaboud FM, Alharby AH (2007). "On Sample Size Estimation for Lomax Distribution." Australian Journal of Basic and Applied Sciences, 1, 373-378.

Abd-Ellah AH (2003). "Bayesian One Sample Prediction Bounds for the Lomax Distribution." Indian Journal of Pure and Applied Mathematics, 30, 101-109.

Abdul-Moniem IB, Abdel-Hameed HF (2012). "On Exponentiated Lomax Distribution." International Journal of Mathematical Archive, 3, 2144-2150.

Adamidis A, Loukas S (1998). "A Lifetime Distribution with Decreasing Failure Rate." Statistics and Probability Letters, 39, 35-42. 
Afify AZ, Cordeiro GM, Maed ME, Alizadeh M, Al-Mofleh H, Nofal ZM (2019). "The Generalized Odd Lindley-G Family: Properties and Applications." Anais da Academia Brasileira de Ciências, 91, 1-22.

Afify AZ, Mohamed OA (2020). "A New Three-Parameter Exponential Distribution with Variable Shapes for the Hazard Rate: Estimation and Applications." Mathematics, 8, 1-17.

Afify AZ, Nassar M, Cordeiro GM, Kumar D (2020). "The Weibull Marshall-Olkin Lindley Distribution: Properties and Estimation." Journal of Taibah University for Science, 14, 192-204.

Al-Awadhi SA, Ghitany ME (2001). "Statistical Properties of Poisson-Lomax Distribution and Its Application to Repeated Accidents Data." Journal of Applied Statistical Science, 10, $365-372$.

Aldahlan M, Afify AZ (2018). "The Odd Exponentiated Half-Logistic Burr XII Distribution." Pakistan Journal of Statistics and Operation Research, 14, 305-317.

Alkasasbeh MR, Raqab MZ (2009). "Estimation of the Generalized Logistic Distribution Parameters: Comparative Study." Statistical Methodology, 6, 262-279.

Ashour SK, Eltehiwy MA (2013). "Transmuted Lomax Distribution." American Journal of Applied Mathematics and Statistics, 1, 21-127.

Barreto-Souza W, Cribari-Neto F (2009). "A Generalization of the Exponential-Poisson Distribution." Statistics and Probability Letters, 79, 2493-2500.

Basu AP, Klein JP (1982). Some Recent Results in Competing Risks Theory Survival Analysis, 216-229. Institute of Mathematical Statistics, Hayward, CA, doi:10.1214/lnms/1215464851. https://projecteuclid.org/euclid.lnms/1215464851.

Cancho VG, Louzada-Neto F, Barriga GDC (2011). "The Poisson-Exponential Lifetime Distribution." Computational Statistics \& Data Analysis, 55, 2493-2500.

Cheng RCH, Amin NAK (1979). Maximum Product of Spacings Estimation with Applications to the Lognormal Distribution. Technical report, Department of Mathematics, University of Wales.

Cheng RCH, Amin NAK (1983). "Estimating Parameters in Continuous Univariate Distributions with a Shifted Origin." J. R. Statist. Soc. B, 45, 394-403.

Cordeiro GM, Afify AZ, Ortega EM, Suzuki AK, Mead ME (2019). "The Odd Lomax Generator of Distributions: Properties, Estimation and Applications." Journal of Computational and Applied Mathematics, 347, 222-237.

Dey S, Ali S, Park C (2015). "Weighted Exponential Distribution: Properties and Different Methods of Estimation." Journal of Statistical Computation and Simulation, 85, 3641-3661.

Dey S, Alzaatreh A, Zhang C, Kumar D (2017a). "A New Extension of Generalized Exponential Distribution with Application to Ozone Data." Ozone: Science $\&$ Engineering, 39, $273-285$.

Dey S, Dey T, Ali S, Mulekar MS (2016). "Two-Parameter Maxwell Distribution: Properties and Different Methods of Estimation." Journal of Statistical Theory and Practice, 10, 291310.

Dey S, Dey T, D K (2014). "Two-Parameter Rayleigh Distribution: Different Methods of Estimation." American Journal of Mathematical and Management Sciences, 33, 55-74. 
Dey S, Kumar D, Ramos PL, Louzada F (2017b). "Exponentiated Chen Distribution: Properties and Estimation." Communication in Statistics - Simulation and Computation, 46, 8118-8139.

Dey S, Nassar M, Kumar D, Alzaatreh A, Tahir MH (2019). "A New Lifetime Distribution with Decreasing and Upside-Down Bathtub-Shaped Hazard Rate Function." STATISTICA, 79, 399-426.

Dey S, Raheem E, Mukherjee S, Ng HKT (2017c). "Two Parameter Exponentiated-Gumbel Distribution: Properties and Estimation with Flood Data Example." Journal of Statistics and Management Systems, 20, 197-233.

Dey S, Zhang C, Asgharzadeh A, Ghorbannezhad M (2017d). "Comparisons of Methods of Estimation for the NH Distribution." Annals of Data Science, 4, 441-455.

Ghitany ME, Al-Awadhi FA, Alkhalfan LA (2007). "Marshal-Olkin Extended Lomax Distribution and Its Application to Censored Data." Communications in Statistics - Theory and Methods, 36, 1855-1866.

Hassan AS, Al-Ghamdi A (2009). "Optimum Step Stress Accelerated Life Testing for Lomax Distribution." Journal of Applied Sciences Research, 5, 2153-2164.

Hassan AS, Nassr SG (2018). "Power Lomax Poisson Distribution: Properties and Estimation." Journal of Data Science, 16, 105-128.

Kao JHK (1958). "Computer Methods for Estimating Weibull Parameters in Reliability Studies." Trans. IRE Reliability Quality Control, 13, 15-22.

Kao JHK (1959). "A Graphical Estimation of Mixed Weibull Parameters in Life Testing Electron Tube." Technometrics, 01, 389-407.

Kumar D, Kumar M (2019). "A New Generalization of the Extended Exponential Distribution with an Application." Annals of Data Sciences, 6, 441-462.

Kundu D, Raqab MZ (2005). "Generalized Rayleigh distribution: Different Methods of Estimations." Computational Statistics and Data Analysis, 49, 187-200.

Kus C (2007). "A New Lifetime Distribution. Computational Statistics and Data Analysis." Computational Statistics and Data Analysis, 51, 4497-4509.

Lee ET, Wang JW (2003). Statistical Methods for Survival Data Analysis. Third Ed. Wiley, New York.

Lemonte AJ, Cordeiro GM (2013). "An Extended Lomax Distribution." Statistics, 47, 800816.

Lomax KS (1954). "Business Failures: Another Example of the Analysis of Failure Data." Journal of the American Statistical Association, 49, 847-852.

Louzada F, Marchi V, Carpenter J (2013). "The Complementary Exponentiated Exponential Geometric Lifetime Distribution." Journal of Probability and Statistics, 2013, Art.ID $502159,12$.

Lu W, Shi D (2012). "A New Compounding Life Distribution: The Weibull Poisson Distribution." Journal of Applied Statistics, 39, 21-38.

Mahmoudi E, Sepahdar A (2013). "Exponentiated Weibull-Poisson Distribution: Model, Properties and Applications." Mathematics and Computers in Simulation, 92, 76-97.

Mathai AM, Haubold HJ (2008). "On Generalized Distributions and Pathways." Physics Letters A, 372, 2109-2113. 
Mazucheli J, Louzada F, Ghitany ME (2013). "Comparison of Estimation Methods for the Parameters of the Weighted Lindley Distribution." Applied Mathematics and Computation, 220, 463-471.

Mead ME (2016). "On Five-Parameter Lomax Distribution: Properties and Applications." Pakistan Journal of Statistics and Operation Research, 12, 185-199.

Mead ME, Afify AZ (2017). "On Five-Parameter Burr XII Distribution: Properties and Applications." South African Statistical Journal, 51, 67-80.

Moors JJA (1988). "A Quantile Alternative for Kurtosis." Journal of the Royal Statistical Society: Series D, 37, 25-32.

Murthy DP, Xie M, Jiang R (2004). Weibull Models. John Wiley Sons.

Nassar M, Afify AZ, Dey S, Kumar D (2018a). "A New Extension of Weibull Distribution: Properties and Different Methods of Estimation." Journal of Computational and Applied Mathematics, 336, 439-457.

Nassar M, Afify AZ, Shakhatreh MK (2020). "Estimation Methods of Alpha Power Exponential Distribution with Applications to Engineering and Medical Data." Pakistan Journal of Statistics and Operation Research, 16, 149-166.

Nassar M, Dey S, Kumar D (2018b). "Logarithm Transformed Lomax Distribution with Applications." Calcutta Statistical Association Bulletin, 70, 122-135.

Ramos MW, Marinho PRD, Silva RV, Cordeiro GM (2013). "The Exponentiated Lomax Poisson Distribution with an Application to Lifetime Data." Advances and Applications in Statistics, 34, 107-135.

Ristic MM, Nadarajah S (2014). "A New Lifetime Distribution." Journal of Statistical Computation and Simulation, 81, 135-150.

Sen S, Afify AZ, Al-Mofleh H, Ahsanullah M (2019). "The Quasi Xgamma-Geometric Distribution with Application in Medicine." Filomat, 33, 5291-5330.

Swain J, Venkatraman S, Wilson J (1988). "Least Squares Estimation of Distribution Function in Johnson's Translation System." Journal of Statistical Computation and Simulation, 29, 271-297.

Tahir MH, Cordeiro GM (2016). "Compounding of Distributions: A Survey and New Generalized Classes." Journal of Statistical Distributions and Applications, 3, 1-35.

Tahir MH, Cordeiro GM, Mansoor M, Zubair M (2015). "The Weibull-Lomax Distribution: Properties and Applications." Hacettepe Journal of Mathematics and Statistics, 44, 461480.

Yousof HM, Afify AZ, Hamedani GG, Aryal G (2017). "The Burr X Generator of Distributions for Lifetime Data." Journal of Statistical Theory and Applications, 16, 288-305.

\section{Affiliation:}

Devendra Kumar

Department of Statistics

Central University of Haryana, Haryana, India

Corresponding author E-mail: devendrastas@gmail.com 


\title{
Mazen Nassar \\ Department of Statistics, Faculty of Science
}

King Abdulaziz University, Jeddah, Saudia Arabia

Department of Statistics, Faculty of Commerce

Zagazig University, Egypt

E-mail: mezo10011@gmail.com

\author{
Ahmed Z. Afify \\ Department of Statistics Mathematics and Insurance, \\ Benha University, Egypt \\ E-mail: ahmed.afify@fcom.bu.edu.eg \\ Sanku Dey \\ Department of Statistics \\ St. Anthony's College, Shillong-793001, Meghalaya, India \\ E-mail: sankud66@gmail.com
}

\section{Austrian Journal of Statistics}

published by the Austrian Society of Statistics

Volume 50

July 2021 http://www.ajs.or.at/

http://www.osg.or.at/

Submitted: 2019-11-05

Accepted: 2020-04-28 